\title{
XML-BSPM: an XML format for storing Body Surface Potential Map recordings
}

\author{
Raymond R Bond*, Dewar D Finlay ${ }^{\dagger}$, Chris D Nugent ${ }^{\dagger}$ and George Moore ${ }^{\dagger}$
}

\begin{abstract}
Background: The Body Surface Potential Map (BSPM) is an electrocardiographic method, for recording and displaying the electrical activity of the heart, from a spatial perspective. The BSPM has been deemed more accurate for assessing certain cardiac pathologies when compared to the 12-lead ECG. Nevertheless, the 12-lead ECG remains the most popular ECG acquisition method for non-invasively assessing the electrical activity of the heart. Although data from the 12-lead ECG can be stored and shared using open formats such as SCP-ECG, no open formats currently exist for storing and sharing the BSPM. As a result, an innovative format for storing BSPM datasets has been developed within this study.

Methods: The XML vocabulary was chosen for implementation, as opposed to binary for the purpose of human readability. There are currently no standards to dictate the number of electrodes and electrode positions for recording a BSPM. In fact, there are at least 11 different BSPM electrode configurations in use today. Therefore, in order to support these BSPM variants, the XML-BSPM format was made versatile. Hence, the format supports the storage of custom torso diagrams using SVG graphics. This diagram can then be used in a 2D coordinate system for retaining electrode positions.

Results: This XML-BSPM format has been successfully used to store the Kornreich-117 BSPM dataset and the LuX-192 BSPM dataset. The resulting file sizes were in the region of 277 kilobytes for each BSPM recording and can be deemed suitable for example, for use with any telemonitoring application. Moreover, there is potential for file sizes to be further reduced using basic compression algorithms, i.e. the deflate algorithm. Finally, these BSPM files have been parsed and visualised within a convenient time period using a web based BSPM viewer.

Conclusions: This format, if widely adopted could promote BSPM interoperability, knowledge sharing and data mining. This work could also be used to provide conceptual solutions and inspire existing formats such as DICOM, SCPECG and aECG to support the storage of BSPMs. In summary, this research provides initial ground work for creating a complete BSPM management system.
\end{abstract}

\section{Background}

\section{The Body Surface Potential Map}

The Body Surface Potential Map (BSPM) is a specialised electrocardiographic method, for recording and displaying the electrical activity of the heart, from a spatial perspective. The BSPM has been deemed more accurate for diagnosing certain cardiac pathologies, when compared

\footnotetext{
* Correspondence: bond-r@email.ulster.ac.uk

${ }^{1}$ Computer Science Research Institute, School of Computing and Mathematics, University of Ulster, Shore Road, Newtownabbey, Co. Antrim, BT37 OQB, UK

+ Contributed equally

Full list of author information is available at the end of the article
}

to the 12-lead electrocardiogram (ECG) [1,2]. The major advantage of the BSPM is its ability to display electrical information in the spatial domain. This is achieved by placing a large number of electrodes (32-213) around the human torso, whereas the 12-lead ECG utilizes six thoracic electrodes which are subject to a limited anatomical area, namely the precordium [3].

\section{Importance of interoperability}

Interoperability is an important area of research, since it promotes the intercommunication of clinical documents between heterogeneous hospital information systems [4]. 
The predominant driver in promoting interoperability has been the development of open formats for storing clinical information [5]. These open formats can be easily integrated into the Electronic Patient Health Record (EPHR). Moreover, according to Fischer et al. [6], cardiological information is progressively being introduced into the EPHR. This is just one reason why a BSPM format should be created. Although the BSPM has been clinically proven to be more accurate for diagnosing cardiac patients, no work has been undertaken to improve the interoperability of BSPM data. Scientists currently store BSPM datasets in a number of custom formats which include propriety data files (e.g. MATLAB, Map3D) and format specific files (formatted Comma Separated Values).

\section{ECG formats}

A plethora of open formats have been created for storing and transmitting the ECG. These formats have been based around more familiar recording methods such as the 12-lead ECG. The three major industrial ECG formats exist; the Digital Imaging and Communication in Medicine ECG (DICOM-ECG) format; Health Level 7 Annotated ECG (HL7/aECG) and the Standardised Communication Protocol ECG (SCP-ECG) format. Other influential ECG formats include ecgML [7], XML-ECG [8], MFER [9], to name but a few. The following Sections provide a more detailed overview of each of the aforementioned three major formats.

\section{DICOM-ECG}

The DICOM standard, formally known as ACR-NEMA was created by the National Electrical Manufactures Association (NEMA) in 1985 [10,11]. ACR-NEMA evolved as DICOM version 3 in 1993, and became a European standard in 1995. The DICOM format originally stored radiographic raster images, from diagnostic devices such as the X-RAY [12]. DICOM now endeavours to support all diagnostic modalities including the ECG. As a result, DICOM waveform supplement 30 was introduced in the year 2000. This extension enables the storage of raw waveform datasets i.e. blood pressure, audio and the ECG. Within the ECG community, DICOM supplement 30 is also called DICOM-ECG.

\section{HL7}

The Annotated ECG (aECG) format was created in partnership between the Health Level 7 and the US Food and Drug Administration (FDA) in 2001 [13]. It was then accepted as a standard by the American National Standards Institute (ANSI) in 2004. The FDA where collecting a large number of ECGs, submitted by pharmaceutical companies for clinical trials. These ECGs where submitted in various formats, some of which where hard copies that had to be scanned for electronic storage. The aECG format was therefore created to improve the administration tasks of managing such a complicated process. This was the first ECG format based on the eXtensible Markup Language (XML).

\section{SCP-ECG}

The SCP-ECG format is a compressed binary based format that takes advantage of Huffman encoding. In 2002, SCP-ECG became a promotion of the European funded OpenECG consortium [5]. The OpenECG network is a body of people dedicated to the interoperability in digital electrocardiography. According to Chronaki et al., they have at least 464 members [14]. In 2005, the SCP-ECG format became the official European standard for the storage and transmission of ECGs [5,14].

All of the aforementioned ECG formats are non proprietary and are therefore capable of achieving interoperability, whereas many ECG Management Systems (EMS) integrate closed proprietary formats into the EPHR. These closed formats include Unipro, Sifor and MDW [15].

It has been shown that none of the aforementioned ECG formats support the storage and transmission of BSPMs. This is likely to be caused by the fact that current ECG formats specialise in catering for popular ECG acquisition methods, such as the 12-lead ECG. It may also be difficult to create a format that supports both the 12lead ECG and the BSPM, since the requirements for storing either are considerably different. This is illustrated in Table 1.

One of the major issues is that, the methods of recording, processing and displaying BSPMs have not yet been standardised [16]. A BSPM can be recorded using an arbitrary number of electrodes placed at customised anatomical locations. According to Hoekema et al. there are at least 11 international electrode layouts that are in use today, some of which use anywhere between 32 (Lux-32 Anterior BSPM) and 219 (Parma-219 BSPM) electrodes as illustrated in Figure 1[16]. In addition, since the number electrodes in BSPM acquisition has not been dictated by a standards consortium, researchers may have developed their own custom electrode layouts for BSPM acquisition.

In contrast to the BSPM, the 12-lead ECG has been standardised since 1938 [17]. The 12-lead ECG utilizes 10 electrodes, which are positioned at well defined anatomical locations [18]. These include six chest electrodes which are placed at specific well defined landmarks on the precordium. It is these specifics that have made it easy to develop storage formats for the 12-lead ECG. Conversely, it is the lack of specifics, hence versatility in BSPM acquisition that leaves the community with the huge challenge of defining a format that supports all BSPM variations. Out of the existing formats, SCP-ECG 
Table 1: Storage requirements

\begin{tabular}{|c|c|c|}
\hline & 12-lead ECG & BSPM \\
\hline Electrodes & 10 & $32-213$ \\
\hline Bipolar leads & 3 & 0 \\
\hline Unipolar leads & 9 & $\begin{array}{l}32-213 \\
\text { *All unipolar, each electrode has an } \\
\text { associated unipolar lead. }\end{array}$ \\
\hline Electrode positions & Standardized. & $\begin{array}{l}\text { Non-standardized } \\
\text { *Range of different layouts. }\end{array}$ \\
\hline Calculated leads & $\begin{array}{l}\text { Standard calculations for generating limb } \\
\text { leads aVF, aVL, aVR and III. }\end{array}$ & $\begin{array}{l}\text { Non standardized } \\
\text { * Range of limited lead sets, for example the } \\
\text { Lux-32 layout expands to Lux-192 using } \\
\text { coefficients. }\end{array}$ \\
\hline Data & Usually a 10 second recording. & $\begin{array}{l}\text { No set recording time. Some BSPMs store } \\
\text { single beats. }\end{array}$ \\
\hline Formatting requirements & $\begin{array}{l}\text { Requires a strict standard format since the } \\
12 \text {-lead ECG is well standardized. }\end{array}$ & $\begin{array}{l}\text { Requires a versatile format to support a } \\
\text { range of electrode layouts. }\end{array}$ \\
\hline Display & $\begin{array}{l}12 \text { waveforms are usually rendered onto } \\
\text { formatted graph paper, i.e. " } 3 \times 4+1 " \text {. }\end{array}$ & $\begin{array}{l}\text { No standard representation exists. Can be } \\
\text { displayed as a series of scalar traces or as a } \\
\text { contour map. } \\
{ }^{*} \text { Electrode positions must be known to } \\
\text { generate a contour map. }\end{array}$ \\
\hline Diagnostic criteria & Diagnostic criteria have been well defined. & Very little diagnostic criteria exist. \\
\hline Accessibility and cost of equipment & $\begin{array}{l}\text { Very accessible within the healthcare } \\
\text { industry and relatively inexpensive. }\end{array}$ & $\begin{array}{l}\text { Not all hospitals have a BSPM recording } \\
\text { and visualisation system. Equipment can } \\
\text { be expensive and hard to get. }\end{array}$ \\
\hline
\end{tabular}

The difference in configuration and storage requirements between the 12-lead ECG and the BSPM.

supports up to 255 leads. These are, however, predefined leads, i.e. right sided chest leads [19]. The format is therefore not versatile enough to support BSPMs. Likewise, a simple change to the aECG specification would enable the storage of an arbitrary number of leads. Unfortunately, this would still leave the problem of storing the actual electrode positions. Given that the current ECG formats focus on storing 12-lead ECG data, such formats do not retain electrode positions because they are standardised and can be easily observed in clinical literature.

BSPM datasets can be displayed as a contour map, a series of scalar traces or as a set of averaged beats. Waveforms are usually positioned over their associated electrode positions using a simple 2D unrolled torso diagram.
This is illustrated in Figure 2. There is no standard torso diagram in place for displaying BSPMs. Researchers currently draw their own custom torso diagrams for displaying BSPM data. Therefore, the three main challenges in creating a BSPM format are:

1. Supporting custom torso diagrams.

2. Storing an arbitrary number of leads.

3. Storing the associated electrode positions for each lead.

From these three challenges, storing electrode positions is the most challenging and arguably the most important aspect of a BSPM format. Electrode positions are required for clinical reference and for visualising the BSPM data, i.e. contour plotting. 


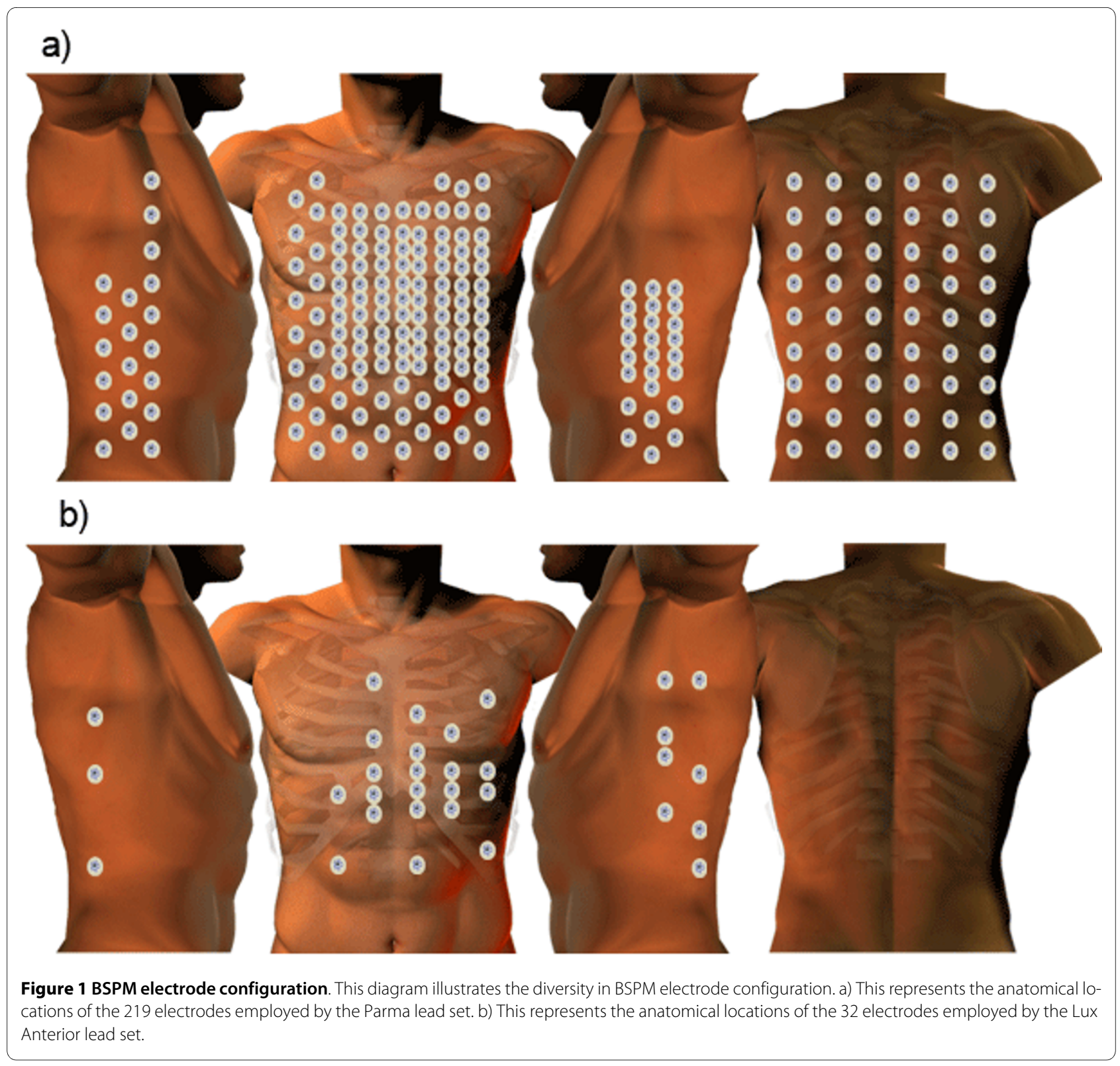

\section{Methods}

There are a number of options when developing a solution to the BSPM storage problem. One option is to propose a BSPM extension to one of the current ECG formats such as SCP-ECG. Another option is to create a new BSPM format. The former option promotes the philosophy of supporting all ECG acquisition methods using a single format. This is similar to the DICOM philosophy, where the aim is to support all diagnostic modalities under one standard. The latter option involves the creation of a specialised format that will only store one ECG acquisition method, namely the BSPM.

Within our current work we have opted to adopt the strategy of creating a specialised format. This decision is partially attributed to the fact that there has been a development in growth in specialised ECG formats. These formats include ecgAware [20], which concentrates on storing ambulatory ECG data, and mECG [21] which concentrates on storing ECG data for mobile devices. Goncalves et al. created the ecgAware format because existing formats such as aECG and ecgML do not support ambulatory ECG monitoring, i.e. Holter monitoring. It is not obvious whether the advantages of general formats like DICOM outweigh the advantages of specific formats such as the ecgAware format. General formats are usually more complex, since they manage a large specification to support a range of modalities, whereas specific formats do not have this complication. Furthermore, since BSPMs 


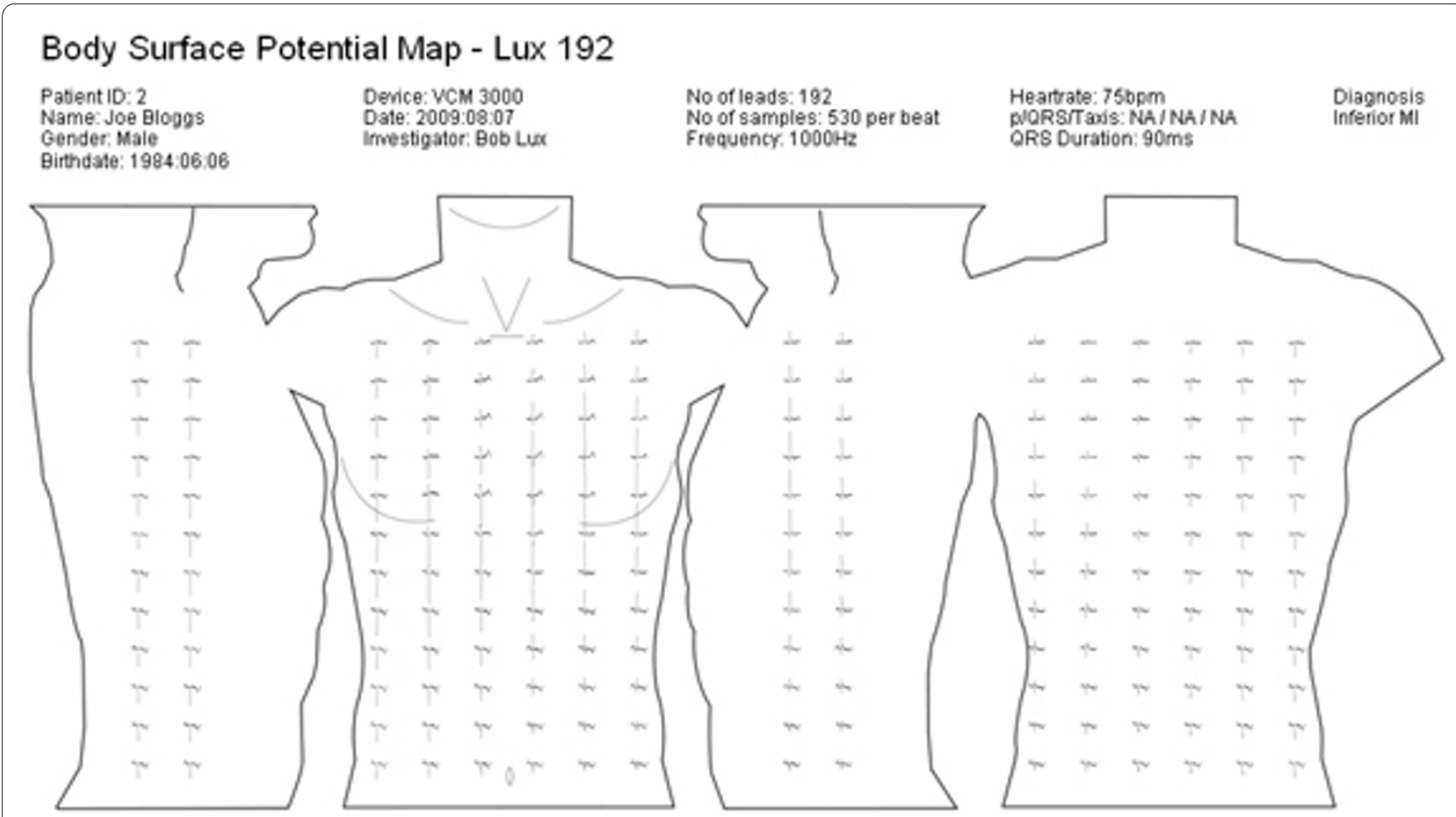

Figure 2 Example BSPM. An unrolled 2D torso diagram displaying 192 averaged beats.

are not as standardised as other ECG acquisition methods, a specialised BSPM format is more appropriate rather than integrating a complex BSPM extension into an existing format. If the proposed BSPM format is not industrialised, then this work, at least, provides the rationale for extending an existing format.

\section{Implementation Method}

$\mathrm{XML}$ and binary are the two predominant implementation methods for storing ECG data. SCP-ECG stores data using a binary based format, whereas the aECG format uses XML. Binary formats are usually smaller in terms of file size, however, disk space and transmission bandwidth requirements are not as much of a major issue as they once were and XML compression techniques do exist [22]. Although XML is more verbose, it does innately benefit from human readability, whereas a computer program is needed to read a binary based format such as SCP-ECG. Unlike binary, XML has the advantage of possibly exploiting a plethora of related XML technologies. These related XML technologies are illustrated in Figure 3. Regarding ECG formats, there has been a growth in XML based formats which may indicate the strength of XML $[7,8,13]$. Moreover, the power of XML within the healthcare industry has been well documented [23]. XML is also promoted by CDISC's Submission Data Model (SDM) [24].
As a result of the aforementioned rationale, an XML based format has been created within this study for storing BSPMs. Figure 4 represents the overall tree structure of this format.

\section{The root element}

The root element bspm stores two required attributes called type and id. The attribute type defines what kind of BSPM data the file is storing. It therefore, indicates to a human observer or a computer program the type of data it is to expect. This is important as there are different methods for recording BSPM data. The type attribute can have one of four values (AVERAGED-BEATS-BSPM, AVERAGED-BEATS-BSPM-TRANSFORM, CONTINUOUS-BSPM or CONTINUOUS -BSPM-TRANSFORM). The value $\boldsymbol{A V E R A G E D - B E A T S - B S P M}$ is used when each lead element within the format stores one single beat as raw number values, whereas the value $\boldsymbol{A} \boldsymbol{V E R}$ AGED-BEATS-BSPM-TRANSFORM indicates that some leads are derived, in that they contain equations as opposed to raw number values. Supporting derived leads is important because there has been substantial research carried out within the ECG community that involves limited lead sets and BSPM transformations [25]. The value CONTINUOUS-BEATS-BSPM, as the name suggests, is used when continuous data is stored for each lead. The value CONTINUOUS-BSPM-TRANSFORM is used to 


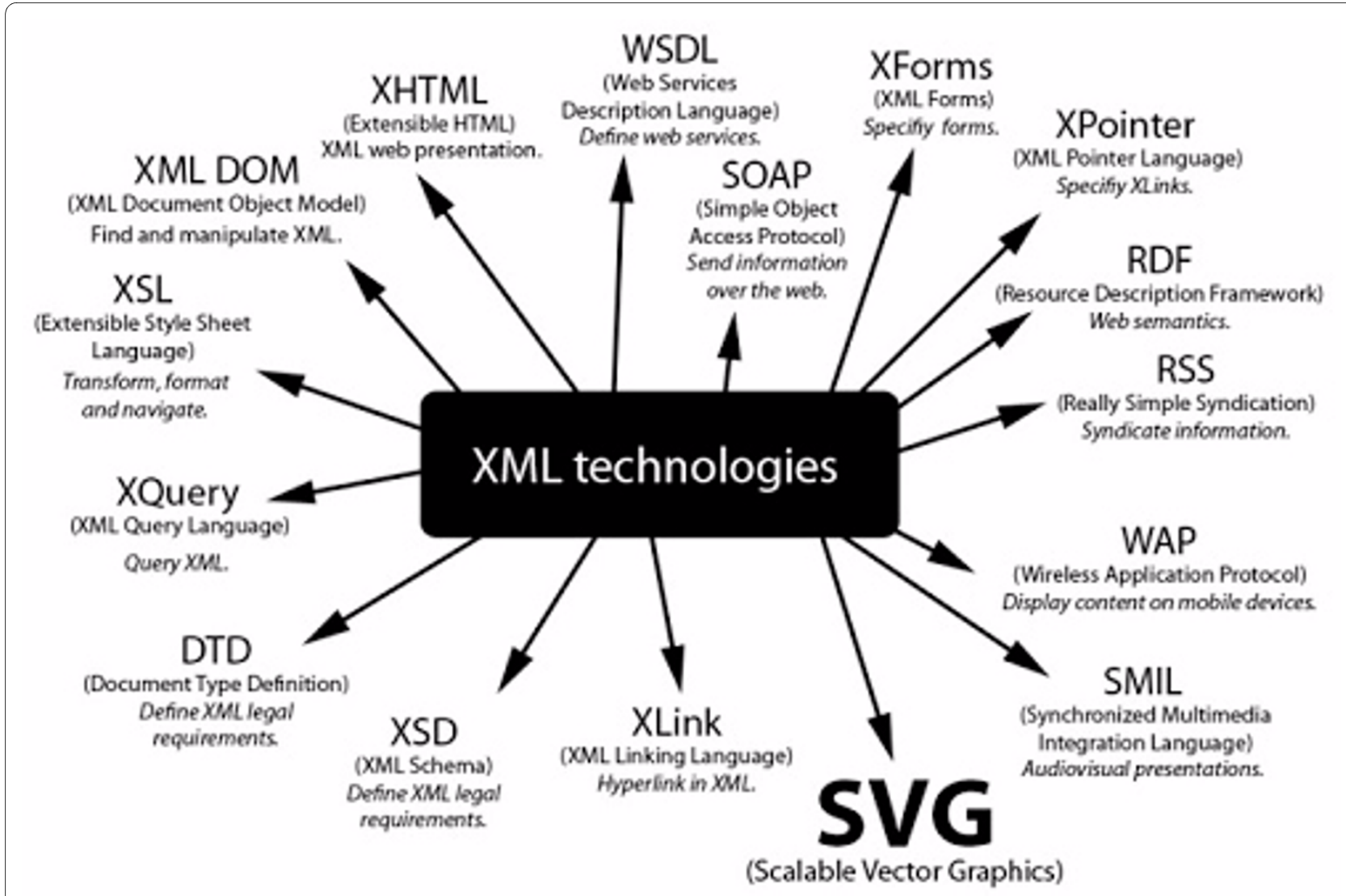

Figure 3 XML technologies. This diagram depicts a plethora of XML related technologies that can be combined with XML-BSPM.

indicate the storage of multiple beats, some of which are stored in raw form and the derived leads are stored as mathematical equations. The attribute $\boldsymbol{i d}$ is used to either uniquely identify a document or to associate the file with a database.

The bspm element has two sub elements called header and leads. The header element stores the metadata. That is, information about the data, which includes patient demographics, record settings, annotations etc. The leads element stores the actual BSPM lead data. A full description of the root element can be seen in Table 2 .

\section{The header element}

The header element does not store any attributes or data of its own. Instead, it is used as a wrapper element to group all of the metadata as sub elements. This separates the metadata from the actual lead data and improves readability of the XML document. The header element can have up to seven sub elements (patient, record, annotations, comments, limbLeads, transformations and diagram) and is described in Table 3.

The patient element is optional given that some BSPM datasets may be associated with a database, which may already retain the patient demographics. In this case, a duplicate would not be necessary. The patient element retains one attribute called $i d$, which can be used to uniquely identify a patient. Moreover, the patient element has a number of sub elements (fullname, sex, DOB, address etc.) as listed in Figure 4. These elements provide the basic information that is needed to fully identify a patient. A full description of the patient element is depicted in Table 4.

The record element is required as it stores important information which is necessary for the visualisation of the data. For example, the frequency of a BSPM recording must be known, in order to draw the actual waveforms. Most of the information, within the record element is stored as attributes, as opposed to elements, as it improves the readability of the XML document and reduces the file size. Figure 5 is an XML excerpt of the record element and illustrates how condensed and readable the record element can be. The record element can have nine attributes, five of which are optional (recordingDevice, recordingTime, recordingDate, investigator, mVSteps) and four that are required (layoutName, leads, samples, frequency). The recordingDevice attribute stores the name of the device that was used for recording the BSPM. This attribute is not required, but 


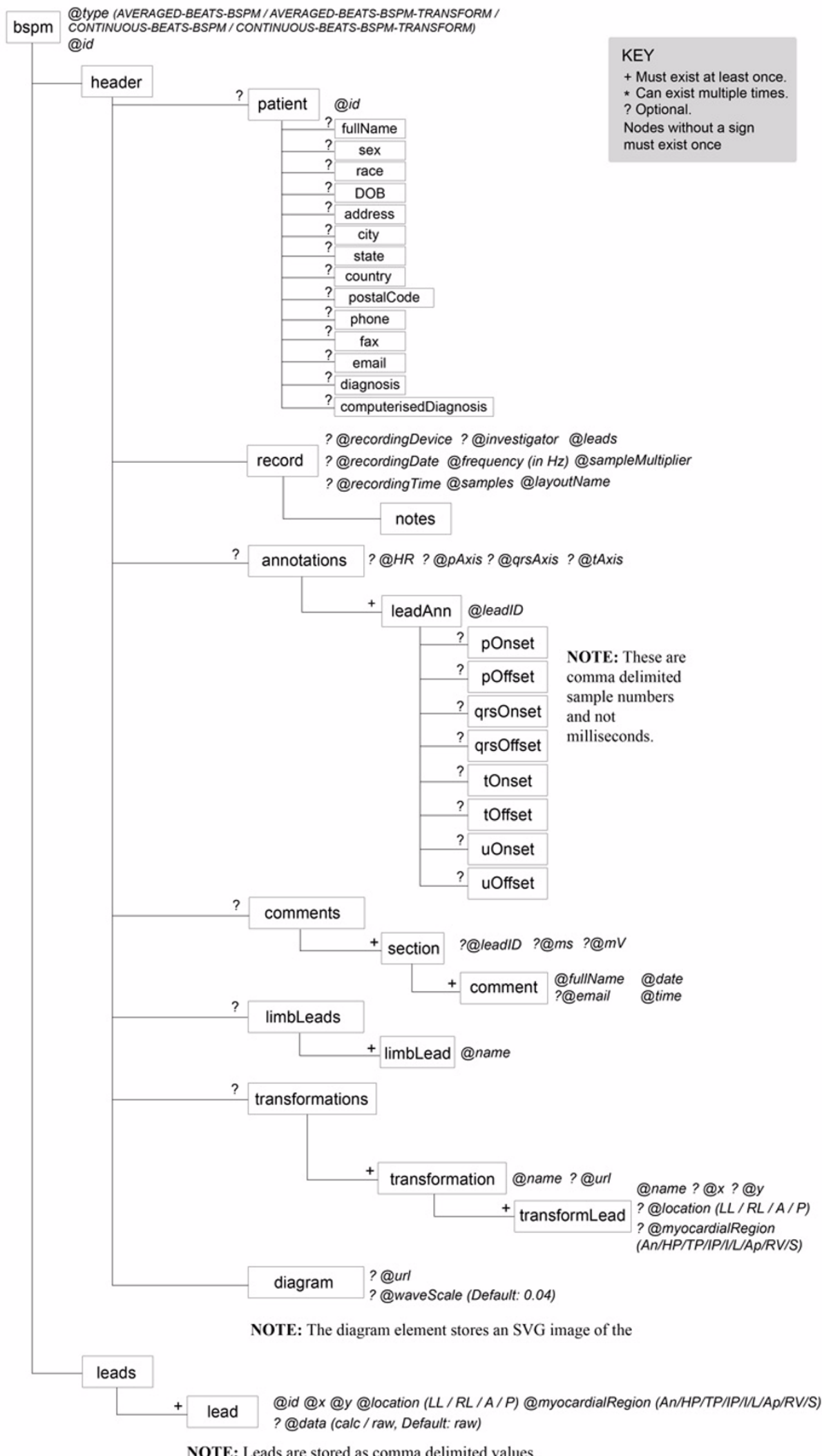

Figure 4 BSPM XML tree structure. Overview of the BSPM XML tree structure. The format is split into two main sections, the header, which retains the meta-data and the leads section, which stores the actual ECG values. 
Table 2: Description of the root element (bspm)

\begin{tabular}{|c|c|c|c|}
\hline \multicolumn{4}{|c|}{ Attributes } \\
\hline Name & Required & Data type & Description \\
\hline type & Required & $\begin{array}{l}\text { AVERAGED-BEATS-BSPM/ } \\
\text { AVERAGED- BEATS -BSPM- } \\
\text { TRANSFORM/CONTINUOUS- } \\
\text { BSPM/CONTINUOUS - BSPM- } \\
\text { TRANSFORM }\end{array}$ & This format can store four types of BSPMs. \\
\hline id & Required & String & For associating the file with a database. \\
\hline \multicolumn{4}{|c|}{ Elements } \\
\hline Name & Required & Data type & Description \\
\hline header & Required & See header table. & $\begin{array}{l}\text { This element separates the data from } \\
\text { metadata, presenting the format in a } \\
\text { coherent fashion. }\end{array}$ \\
\hline leads & Required & See leads table. & $\begin{array}{l}\text { This element groups the entire lead data. It } \\
\text { has been named leads as opposed to } \\
\text { channels because all BSPM leads are } \\
\text { unipolar. }\end{array}$ \\
\hline
\end{tabular}

such information may be helpful when comparing different recording devices. The attributes recordingTime and recordingDate store the time and date of when the actual BSPM recording took place. These are also optional given that this information may have been disregarded. However, the time and date of a diagnostic procedure is important in most clinical scenarios. The investigator attribute stores the full name of the clinician who carried out the procedure. This is an optional attribute due to the fact that procedures cannot be attributed to only one investigator. The layoutName attribute is required as there are many electrode layouts that can be used in a BSPM acquisition. Therefore, it is important to define which electrode layout was used. The leads attribute is required because it stores the number of leads that have been stored within the document. This number excludes any limb leads that have been stored within the header element. The next three attributes (samples, frequency and sampleMultiplier) are important, since their values assist in rendering the actual waveforms. The samples attribute stores the number of sample values that have been stored in each lead. The frequency attribute stores the value of the sample frequency in Hz. Finally, the sampleMultiplier attribute stores a number which is used for mathematically multiplying and hence magnifying each of the sample values. This attribute has been added to support legacy datasets where sample values need to be multiplied by such a number in order to retrieve the actual values. This value is used to improve the resolution of the waveforms. Although this attribute is optional, if it is not present, the value defaults to 1 . The record element can have one sub element, called notes. This element gives the investigator or the technician the option to insert a detailed description of the recording procedure. A full description of the record element can be seen in Table 5 .

The annotations element is optional, but in many cases can be important for post processing and clinical analysis. For example, an ST40 isopotential map is commonly used to assist in diagnosing ischemic disorders. A map such as this cannot be easily generated if the relevant annotations have not been defined within the format. The annotations element can store four optional attributes. The HR attribute is an abbreviation for heart rate. The heart rate can be stored within the format given that a lot of BSPM datasets store one beat and at least two beats are required to calculate the heart rate using the R-R interval. Therefore, this attribute allows the clinician to calculate the heart rate and store it if only single beats are retained. The other attributes pAxis, qrsAxis and tAxis store the cardiac axis in degrees. We have facilitated the storage of cardiac axis as it cannot be easily calculated from the non 
Table 3: Description of the header element

\begin{tabular}{|c|c|c|c|}
\hline Elements & & & \\
\hline Name & Required & Data type & Description \\
\hline patient & Required & See patient table. & $\begin{array}{l}\text { Patient demographics and } \\
\text { diagnosis. }\end{array}$ \\
\hline record & Required & See record table. & $\begin{array}{l}\text { Information about the } \\
\text { recording settings, i.e. device, } \\
\text { time, and recording physician } \\
\text { etc. }\end{array}$ \\
\hline annotations & Required & See annotations table. & $\begin{array}{l}\text { This is a wrapper element for } \\
\text { storing beat markers, i.e. } p \\
\text { onset. }\end{array}$ \\
\hline comments & Optional & See comments table. & $\begin{array}{l}\text { This can be used for } \\
\text { collaboration and discussion } \\
\text { amongst clinicians. }\end{array}$ \\
\hline limbLeads & Optional & See limbLeads table. & $\begin{array}{l}\text { Some BSPM datasets retain } \\
\text { the limb leads that were used } \\
\text { to calculate the Wilson Central } \\
\text { Terminal (WCT). These limb } \\
\text { leads can be stored here and } \\
\text { may prove useful in post } \\
\text { processing. }\end{array}$ \\
\hline transformations & Optional & See transformations table. & $\begin{array}{l}\text { This is where equations can be } \\
\text { stored to transform the BSPM } \\
\text { into the } 12 \text {-lead ECG or the } \\
\text { VCG. }\end{array}$ \\
\hline diagram & Required & $\begin{array}{l}\text { CDATA, see diagram table for } \\
\text { attributes. }\end{array}$ & $\begin{array}{l}\text { This element stores an SVG } \\
\text { diagram that represents an } \\
\text { unrolled } 2 \mathrm{D} \text { torso. }\end{array}$ \\
\hline
\end{tabular}

standard leads that often make up a BSPM. The annotations element has one sub element, namely leadAnn. The leadAnn element stands for lead annotation. This element must exist at least once, but can also exist multiple times. A description of the annotations element can be seen in Table 6.

The leadAnn element acts as a wrapper tag that groups all of the beat markers, i.e.pOnset, pOffset etc. The lead$A n n$ element has one required attribute called leadID. This leadID attribute stores a value that corresponds with the $\boldsymbol{i d}$ attribute of the lead element. This means, each lead can have its own independent beat markers. However, most BSPM datasets use the same beat markers for all the leads. If this is the case, the value "*" can be ascribed to the leadID attribute. This value is commonly used in computer science to select all the items in concern. Therefore in this context, the value "*: would instruct a computer program to use the defined beat markers for all leads. The leadAnn element can have eight sub elements ( $p$ Onset, $p$ Offset, qrsOnset, qrsOffset etc). Each sub element stores sample values that define beat markers, respectively. For example, the pOnset element stores sample numbers that refer to the $\mathrm{p}$ wave onset locations. These eight sub elements will each store a single value if the BSPM being stored is a set of averaged single beats. On the contrast, multiple values are stored in a CSV format if the BSPM being stored is a set of multiple beats. A full description of the leadAnn element can be seen in Table 7 . 
Table 4: Description of the patient element

\begin{tabular}{|c|c|c|c|}
\hline Attributes & & & \\
\hline Name & Required & Data type & Description \\
\hline id & Required & String & $\begin{array}{l}\text { This is used to uniquely identify a } \\
\text { patient, e.g. } 0000001 \text {. }\end{array}$ \\
\hline \multicolumn{4}{|l|}{ Elements } \\
\hline Name & Required & Data type & Description \\
\hline fullName & Optional & String & Name of the patient, e.g. John Smith. \\
\hline sex & Optional & $\begin{array}{l}\text { male/female/ } \\
\text { unspecified/unknown }\end{array}$ & E.g. male. \\
\hline DOB & Optional & Date YYYY-MM-DD & E.g. 1984-06-24. \\
\hline address & Optional & String & E.g. 47 University street \\
\hline city & Optional & String & E.g. Belfast \\
\hline state & Optional & String & E.g. Antrim \\
\hline country & Optional & String & E.g. Northern Ireland \\
\hline postalCode(ZipCode) & Optional & String & $\begin{array}{l}\text { This element can store either a } \\
\text { postcode or a Zipcode, e.g. BT37 0QB. }\end{array}$ \\
\hline phone & Optional & String & $\begin{array}{l}\text { The data type is string to support } \\
\text { commas and hyphens, e.g. } 08700400 \\
700 .\end{array}$ \\
\hline fax & Optional & String & E.g. 08700400700. \\
\hline email & Optional & String & E.g.johnsmith@myemailhost.com \\
\hline diagnosis & Optional & String & $\begin{array}{l}\text { This element is for storing the } \\
\text { patient's diagnosis, as perceived by a } \\
\text { clinician, e.g. Acute Myocardial } \\
\text { Infarction. }\end{array}$ \\
\hline computerisedDiagnosis & Optional & String & $\begin{array}{l}\text { This element is used to record the } \\
\text { predicted diagnosis made by a } \\
\text { computerised classification algorithm, } \\
\text { e.g. Left Bundle Branch Block. }\end{array}$ \\
\hline
\end{tabular}


Table 5: Description of the record element

\begin{tabular}{|c|c|c|c|}
\hline Attributes & & & \\
\hline Name & Required & Data type & Description \\
\hline recordingDevice & Optional & String & $\begin{array}{l}\text { The name of the device used } \\
\text { to record the BSPM, e.g. VCM- } \\
3000 \text {. }\end{array}$ \\
\hline recordingTime & Optional & Time:HH:MM:SS:SSS & $\begin{array}{l}\text { The time of day the recording } \\
\text { took place, e.g. 12:50:00:000. }\end{array}$ \\
\hline recordingDate & Optional & Date YYYY-MM-DD & $\begin{array}{l}\text { The date the recording took } \\
\text { place, e.g. } 2009-08-28 \text {. }\end{array}$ \\
\hline investigator & Optional & String & $\begin{array}{l}\text { The name of the recording } \\
\text { clinician, e.g. Fred Kornreich. }\end{array}$ \\
\hline layoutName & Required & String & $\begin{array}{l}\text { The name of BSPM } \\
\text { configuration method, e.g. } \\
\text { Lux-192. }\end{array}$ \\
\hline leads & Required & Integer & $\begin{array}{l}\text { This records the number of } \\
\text { leads that have been stored } \\
\text { within the leads element, e.g. } \\
\text { 192. }\end{array}$ \\
\hline samples & Required & Integer & $\begin{array}{l}\text { This stores the number of } \\
\text { sample values that have been } \\
\text { stored for each lead, e.g.600. }\end{array}$ \\
\hline frequency & Required & String & $\begin{array}{l}\text { Number of samples per } \\
\text { second recorded in Hertz, e.g. } \\
\text { "1000 Hz" can be interpreted } \\
\text { as } 1000 \text { samples per second. }\end{array}$ \\
\hline sampleMultiplier & Optional & Float DEFAULT: 1 & $\begin{array}{l}\text { This is the number each } \\
\text { sample value must be } \\
\text { multiplied by, in order to get } \\
\text { the actual value. If this } \\
\text { attribute does not exist, the } \\
\text { value defaults to } 1 .\end{array}$ \\
\hline \multicolumn{4}{|l|}{ Elements } \\
\hline Name & Required & Data type & Description \\
\hline notes & Optional & String & $\begin{array}{l}\text { This allows the investigator to } \\
\text { include notes that best } \\
\text { describe the recording } \\
\text { procedure. }\end{array}$ \\
\hline
\end{tabular}




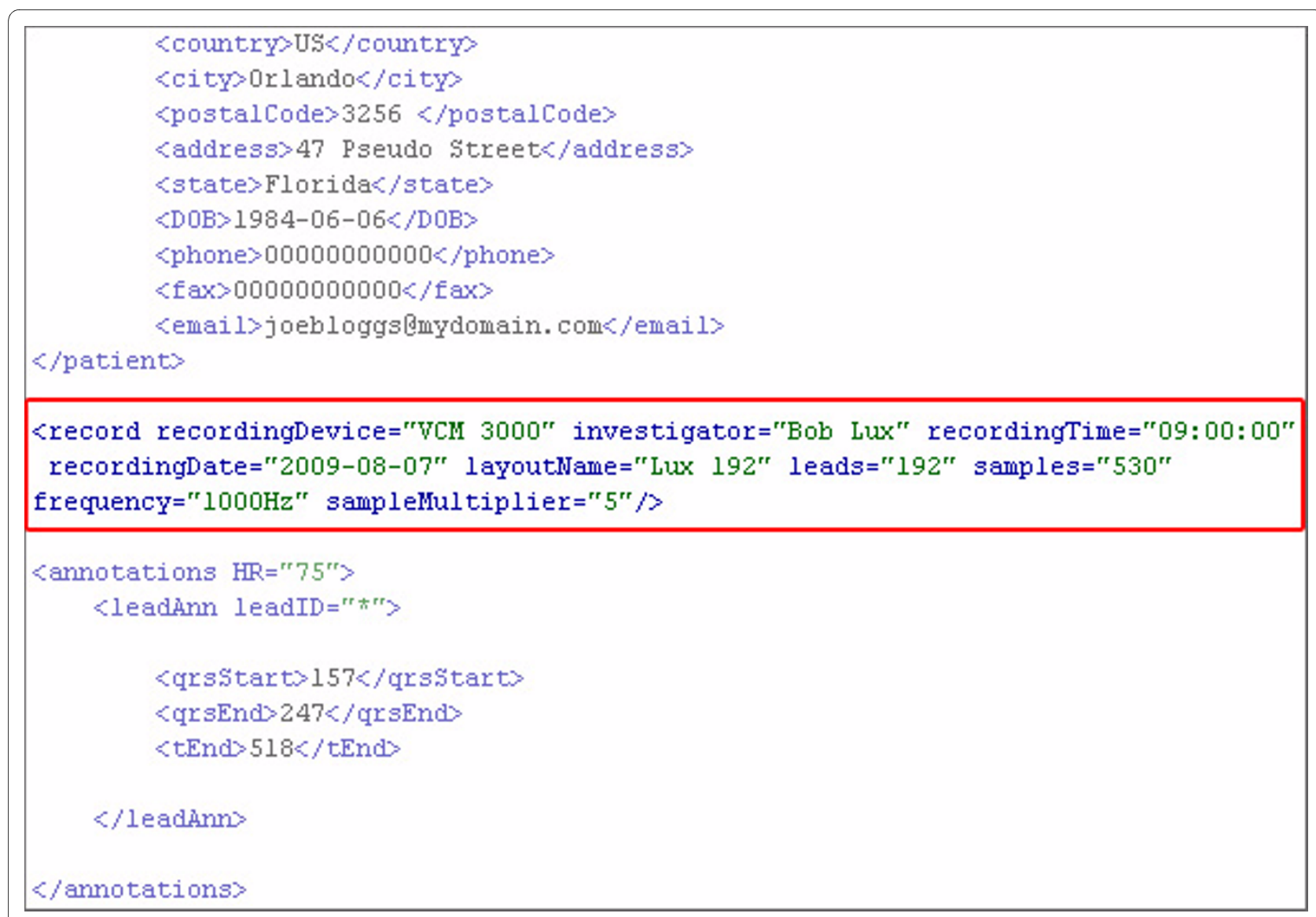

Figure 5 record element. An XML excerpt of the record element, illustrating the readability of its attributes.

The comments element allows an observer or multiple observers to store textual information alongside the waveform data. This feature can be used for collaboration and discussion, since evaluating a BSPM is still a relatively new process and is not, as yet, a fine art. The comments element does not have any attributes, but it has one sub element called section. This element can exist multiple times within the comments element. The section element is used to group comments that refer to the same section of the same lead. This way, comments can be formatted and presented similarly to online forums. The section element has three optional attributes. The leadID attribute makes reference to a particular lead within the leads element by storing the id of a lead. If a comment is being referred to the whole BSPM, the "*" can be attributed to the leadID attribute. The attributes $\boldsymbol{m s}$ (milliseconds) and $\boldsymbol{m} \boldsymbol{V}$ (microvolts) are also optional. These attributes specify which particular portion of a lead; the enclosed comment(s) are referring to. The section element has one sub element called comment, which must exist at least once. This element stores the actual textual information inserted by a clinician. A full description of the section element can be viewed in Table 8 .
The comment element has four attributes. Three of these are required (fullName, date, time) and the other one is optional (email). The fullName attribute is required because the source of a comment is important and this is how most people assess the credibility of an observation. The date and time attributes are required, so that comments can be presented chronologically. The email attribute can either be used for identification or for correspondence. It is, however, optional since every user may not have or agree in submitting their email address. A full description of the comment element can be found in Table 9. Also, Figure 6 illustrates how this comments feature could be presented within a BSPM viewing application.

The limbLeads element is used to store any limb leads that were recorded to calculate the WCT. This element is optional since not all BSPM datasets retain limb lead data. Nevertheless, retaining limb lead data may prove useful for post processing. This element has one sub element called limbLead which can appear multiple times. The limbLead element has one required attribute called name, which stores the name of the limb lead, i.e. I, II etc. Legacy limb leads can also be stored such as the VF, VR 
Table 6: Description of the annotations element

\begin{tabular}{|c|c|c|c|}
\hline \multicolumn{4}{|c|}{ Attributes } \\
\hline Name & Required & Data type & Description \\
\hline$H R$ & Optional & Integer & $\begin{array}{l}\text { The Heart Rate can be stored, } \\
\text { because a patient's heart rate } \\
\text { can not be calculated from a } \\
\text { single beat, e.g. } 70 \text { bpm. }\end{array}$ \\
\hline pAxis & Optional & Integer & In degrees, e.g. $40^{\circ}$. \\
\hline qrsAxis & Optional & Integer & In degrees, e.g. $70^{\circ}$. \\
\hline tAxis & Optional & Integer & In degrees, e.g. $50^{\circ}$. \\
\hline \multicolumn{4}{|l|}{ Elements } \\
\hline Name & Required & Data type & Description \\
\hline leadAnn & Required & See leadAnn table. & $\begin{array}{l}\text { leadAnn stands for lead } \\
\text { annotation. }\end{array}$ \\
\hline
\end{tabular}

and VL leads given that legacy datasets and in particular the Kornreich-117 dataset [26] retains these limb leads. The limbLeads element and sub elements are described in Table 10.

The transformations element can be used to store any required equations required for further post processing. For example, it could be used to store equations for extracting the 12-lead ECG or the vectorcardiogram (VCG) from the BSPM dataset. Another example would be to store equations that could transform the BSPM into another BSPM dataset [27]. The transformations element has one optional sub element called transformation, which may appear multiple times. A description of the transformations element can be seen in Table 11.

The transformation element as described in Table 12 has one sub element called transformLead, which can appear multiple times. It also contains one required attribute called name, which stores the actual name of the transformation, for example, 12-lead ECG. The transformation element has another optional attribute called $\boldsymbol{u r l}$, which is used to retain a link to an XML file containing the actual calculations. By using this $\boldsymbol{u r l}$ attribute, BSPM files can share the same transformation equations. The transformLead element has one required attribute (name) and four optional attributes $(x, y$, location, myocardialLocation). These four attributes have been borrowed from the main lead element. They are optional here given that such information is not always relevant to every transformation. The name attribute is mandatory as it stores either the lead number or the lead name, i.e. $\boldsymbol{a} \boldsymbol{V F}$. Finally, the transformLead element stores the actual equation for calculating a lead. A description of the transformLead element can be found in Table 13.

The rationale for storing equations in a BSPM format is that there are many custom electrode layouts. Therefore, each layout warrants its own unique set of equations for deriving, for example, the 12-lead ECG. Such calculations are also required as BSPM configurations do not usually contain the 12-lead ECG precordial electrode positions as a subset. Nevertheless, there is at least one BSPM lead configuration which has the six precordial leads as a subset of the BSPM leads [28], but most BSPM systems do not include all six precordial leads.

The main challenge when creating the transformLead element was defining a method for storing equations. One can define an equation using normal mathematical syntax; however, a simple method has been set in place for referring to individual BSPM leads. This method can be seen in Figure 7. This diagram illustrates a simple two lead BSPM that has been stored using this XML format. Suppose that these two leads where recorded from the precordial region and that an imaginary V1 lead from a 12-lead ECG could be positioned midway between the two BSPM leads. If this was the case, then a pseudo V1 lead could be calculated by storing an equation within the transformLead element. This is illustrated in section D 
Table 7: Description of the leadAnn element

\begin{tabular}{|c|c|c|c|}
\hline \multicolumn{4}{|c|}{ Attributes } \\
\hline Name & Required & Data type & Description \\
\hline leadID & Required & String & $\begin{array}{l}\text { This value corresponds to the } \\
\text { id attribute stored within each } \\
\text { lead element. This attribute is } \\
\text { used to identify which lead } \\
\text { the beat markers are referring } \\
\text { to. The value * means that the } \\
\text { beat markers refer to all the } \\
\text { leads. }\end{array}$ \\
\hline
\end{tabular}

\begin{tabular}{|c|c|c|c|}
\hline Elements & & & \\
\hline Name & Required & Data type & Description \\
\hline pOnset & Optional & String & $\begin{array}{l}\text { Stores the onset of the } P \\
\text { deflection. Multiple values are } \\
\text { stored as CSV. }\end{array}$ \\
\hline pOffset & Optional & String & $\begin{array}{l}\text { Stores the offset of the } P \\
\text { deflection. Multiple values are } \\
\text { stored as CSV. }\end{array}$ \\
\hline qrsOnset & Optional & String & $\begin{array}{l}\text { Stores the onset of the QRS } \\
\text { deflection. Multiple values are } \\
\text { stored as CSV. }\end{array}$ \\
\hline qrsOffset & Optional & String & $\begin{array}{l}\text { Stores the offset of the QRS } \\
\text { deflection. Multiple values are } \\
\text { stored as CSV. }\end{array}$ \\
\hline tOnset & Optional & String & $\begin{array}{l}\text { Stores the onset of the T } \\
\text { deflection. Multiple values are } \\
\text { stored as CSV. }\end{array}$ \\
\hline tOffset & Optional & String & $\begin{array}{l}\text { Stores the offset of the T } \\
\text { deflection. Multiple values are } \\
\text { stored as CSV. }\end{array}$ \\
\hline uOnset & Optional & String & $\begin{array}{l}\text { Stores the onset of theU } \\
\text { deflection. Multiple values are } \\
\text { stored as CSV. }\end{array}$ \\
\hline uOffset & Optional & String & $\begin{array}{l}\text { Stores the offset of the } U \\
\text { deflection. Multiple values are } \\
\text { stored as CSV. }\end{array}$ \\
\hline
\end{tabular}


Table 8: Description of the section element

\begin{tabular}{|c|c|c|c|}
\hline Attributes & & & \\
\hline Name & Required & Data type & Description \\
\hline leadID & Optional & String & $\begin{array}{l}\text { This attribute is used to } \\
\text { specify which lead the sub } \\
\text { comments refer to. If a } \\
\text { comment refers to all leads, } \\
\text { the value "*" can be used. }\end{array}$ \\
\hline ms & Optional & Float & $\begin{array}{l}\text { This ms (milliseconds) } \\
\text { attribute specifies which part } \\
\text { of the waveform the sub } \\
\text { comments are referring to. }\end{array}$ \\
\hline$m V$ & Optional & Float & $\begin{array}{l}\text { The voltage level at which the } \\
\text { sub comments are referring } \\
\text { to. }\end{array}$ \\
\hline \multicolumn{4}{|l|}{ Elements } \\
\hline Name & Required & Data type & Description \\
\hline Comment & optional & $\begin{array}{l}\text { String, see comment table for } \\
\text { attributes }\end{array}$ & $\begin{array}{l}\text { Comments can be used for } \\
\text { collaboration and knowledge } \\
\text { sharing amongst researchers } \\
\text { and/or clinicians. }\end{array}$ \\
\hline
\end{tabular}

of Figure 7. This is a simple mean equation that adds each value from leads one and two and averages them by dividing them by two. This equation could be represented within the transformLead element as "([Lead1] + [Lead2])/2". The square brackets encompassing the word 'Lead' followed by the actual BSPM lead number is the syntactical method used within this format to reference a BSPM lead in an equation.

The diagram element is a required sub element of the header tag. It is required as it stores an unrolled torso schematic that is used as a reference diagram in a $2 \mathrm{D}$ coordinate system. This $2 \mathrm{D}$ coordinate system is used for storing electrode positions relative to the torso. This format must store electrode positions, in order to support different BSPM electrode layouts. Knowledge of electrode positions is also useful for clinical reference and visualizing the BSPM, i.e. contour plotting. Interestingly, like the BSPM, the number of electrodes used in certain instances to record an electroencephalogram (EEG) is not as standardised. These electrodes can also be positioned at custom cranial landmarks. Therefore, since the EEG and the BSPM are not as standardised as the 12-lead ECG, some EEG formats such as the Extensible Biosignal
Format (EBS) store the actual electrode positions. The EBS format was created in 1993 by Marcus Kuhn and the specification is freely available online [29]. The EBS format stores the electrode positions within the header of the file using a Computer Graphics Metafile (CGM). These EEG formatting concepts provided inspiration in the development of the proposed BSPM format.

The diagram element has two optional attributes called $\boldsymbol{u r l}$ and waveScale. The $\boldsymbol{u r l}$ attribute is an abbreviation for Uniform Resource Locator (URL). This attribute stores a path or a web address to a torso diagram, which can reside either on the internet or on a local network. The waveScale attribute stores a float value between zero and one. This value is used to proportionally scale BSPM leads (scalar traces) to fit comfortably in their associated electrode positions, in relation to the size of the torso diagram. For example, the value 0.1 would scale the waveforms to 10 percent of their actual size. Although this attribute is optional, if it does not exist then the value defaults to 0.04 , which scales all waveforms to $4 \%$ of their actual size. This default value has been chosen, as it proportionally scales waveforms in relation to torso dia- 
Table 9: Description of the comment element

\begin{tabular}{|c|c|c|c|}
\hline Attributes & & & \\
\hline Name & Required & Data type & Description \\
\hline fullName & Required & Float & $\begin{array}{l}\text { Storing the full name is useful } \\
\text { for tracking discussions, e.g. } \\
\text { Dr. John Smith. }\end{array}$ \\
\hline email & Optional & Float & $\begin{array}{l}\text { Email can be used for both } \\
\text { identification and } \\
\text { correspondence. }\end{array}$ \\
\hline date & Required & Date YYYY-MM-DD & E.g. 12:50:00:000. \\
\hline time & Required & Time:HH:MM:SS:SSS & E.g. 2009-08-28. \\
\hline
\end{tabular}

grams that are approximately the size of an A4 piece of paper. This is a standard paper size within the UK.

The advantage of implementing a format in XML as opposed to binary is its accessibility to a suite of related XML technologies, i.e. Scalable Vector Graphics (SVG). SVG is a W3C recommendation and an XML language for describing images [30]. The diagram element stores an unrolled 2D torso schematic using SVG. This element is similar to the idea suggested in another study [31], which is to integrate a photograph of the subject into the format, in order to retain electrode locations.

Raster images bulk up the size of the file and a photograph does not give direct programmatic access. SVG images scale better and are smaller in terms of file size when compared to rasterised equivalents. An example of a simple SVG torso diagram is depicted in Figure 8a[16]. This particular SVG diagram is just one kilobyte in size and 400 bytes when compressed (ZIP), whereas the rasterised (JPEG) equivalent is 50 kilobytes. As of yet, there has been no standard torso schematic proposed for displaying BSPMs. As previously mentioned, currently researchers draw their own custom torso diagrams, which is why the proposed BSPM format allows for the integration of custom torso diagrams using SVG. Although Figure 8a is a basic torso diagram, SVG has the ability to describe complex torsos such as the one depicted in Figure 8b. Unfortunately, intricate diagrams usually mean larger file sizes.

There are three options when integrating an SVG diagram into the BSPM format. Method one is to embed SVG markup directly into the diagram element. The disadvantage is that, a large embedded SVG diagram will increase the size of the document and make it less readable. The advantage of this method is that, it excludes the need for network or internet access for loading an external SVG file. Method two is to reference a URL using the url attribute, which stores a path to an SVG diagram. This method does reduce the size of the file; however, it relies on external access to an SVG file. If the external file no longer exists, then the BSPM file becomes redundant, since a diagram is required to establish the electrode positions. This method is also disadvantageous as multifile management can be a problem [8]. Finally, method three combines both the previous methods and has been adopted in the current work. This approach stores a URL linking to a complex SVG torso diagram, i.e. a torso outline with anatomical structures such as a rib cage, whilst embedding a simplistic version of that diagram. In effect, when a computer program cannot load the external complex SVG file, it can alternatively load the simplistic embedded version. Therefore, method three keeps the file size at a minimum whilst retaining the capability of loading a sophisticated torso diagram. Method three is also advantageous because it can always retain its electrode positions. Even if the external SVG file is lost, it utilises the embedded version in its place. An example of method three can be viewed in Figure 9. The data type for the diagram element is Character Data (CDATA). CDATA allows the element to store XML markup without it being processed. This means that web browsers will display the BSPM format in the normal XML tree structure. Otherwise, without CDATA as the data type, some web browsers would disrupt the tree structure by rendering the SVG diagram on top of the XML. Also, the absence of the CDATA data type would complicate the format and demand namespace management. A description of the diagram element can be found in Table 14. 


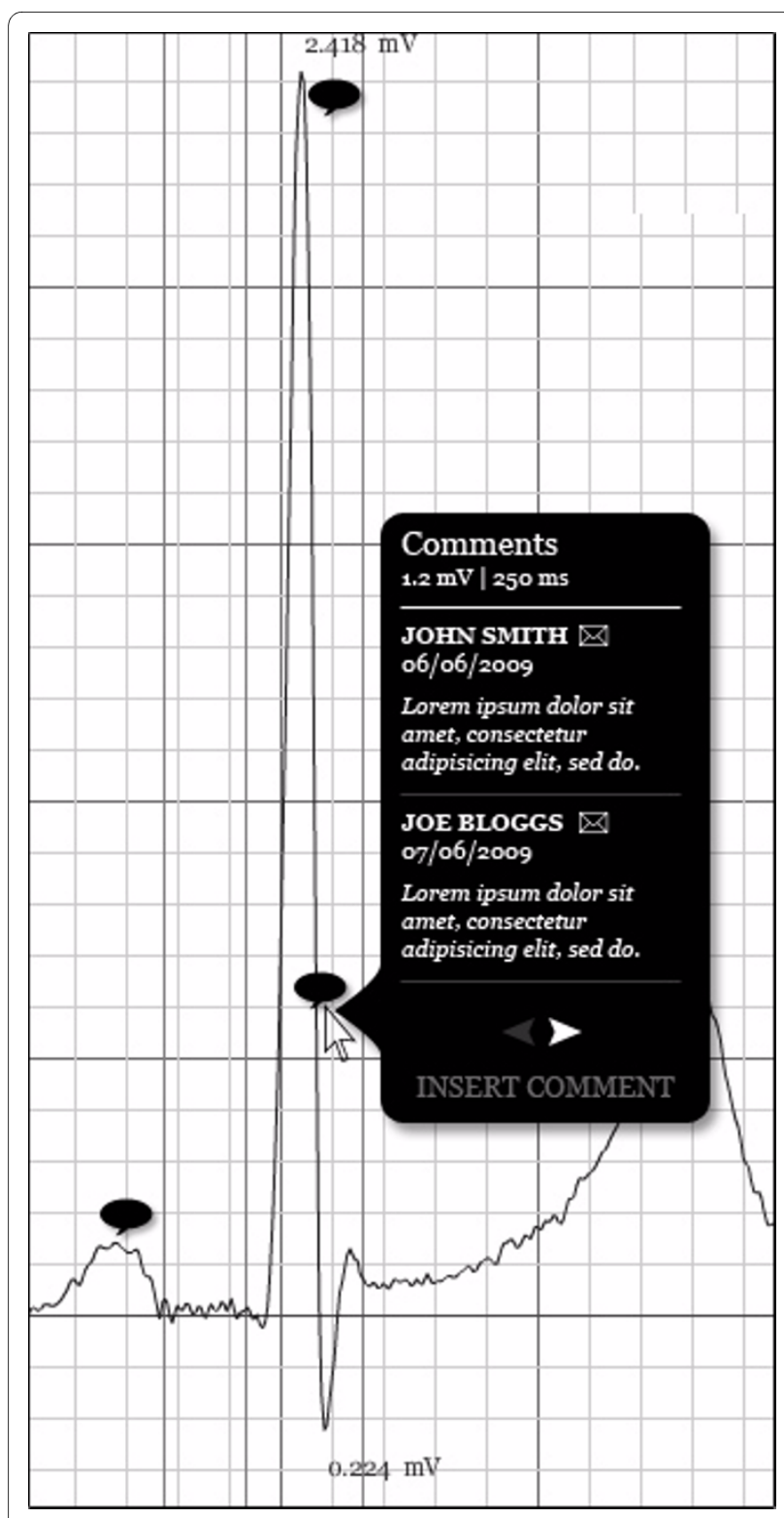

Figure 6 Representing comments. A mock-up of how the comments feature could be presented in a BSPM viewing system.
The leads element is a sub element of the root element, bspm. This element acts as a wrapper tag that accumulates all the subordinate BSPM leads. Hence, the leads element has one sub element called lead, which can appear multiple times. As its name suggests, each lead element stores a unipolar ECG lead. Other ECG formats store lead data using a different name, i.e. aECG [13] uses the name 'digits' and the ecgML [7] format uses the name 'channel'.

The lead element as described in Table 15 has six attributes, three of which are optional and three that are required. The $\boldsymbol{i d}$ attribute is a requirement, since it simply stores the lead number. For example, to store the Lux192 BSPM dataset, there would have to be 192 lead elements within the leads element. The $\boldsymbol{x}$ and $\boldsymbol{y}$ attributes are also required. These attributes store numerical pixel values which are, in effect, 2D coordinate values. These coordinate values are used to derive electrode positions, which is performed with reference to the SVG torso schematic that is stored within the diagram element. This 2D coordinate system for storing electrode positions is demonstrated in Figure 10.

The location attribute is optional and is used to define which portion of the torso, the electrode was positioned. This attribute can have four possible abbreviated values, i.e. LL (Left Lateral), RL (Right Lateral), A (Anterior) and $\mathrm{P}$ (Posterior). Defining the general location of each electrode, allows a computer program to best visualise and distinguish between leads, for example, anterior leads could be rendered a different colour from the posterior leads. The myocardialRegion attribute is used to define the corresponding region of the myocardium each lead refers to. The nine possible values are abbreviated, i.e. Ap stands for apical. Using this attribute, a computer program can assess ST elevation in the appropriate leads for diagnosing acute myocardial infarction. Finally, the data attribute is also optional and is used to define whether the content of the lead element contains raw CSV values or an equation, that when executed calculates lead data. Hence, the data attribute can only have one of two values, i.e. raw or calc (calculation). The raw value indicates that

Table 10: Description of the limbLeads element Elements

\begin{tabular}{llll}
\hline Name & Required & Data type & Description \\
\hline limbLead & Required & String & Stores limb lead data. Multiple values are stored as CSV.
\end{tabular}

Attributes(for limblead element)

\begin{tabular}{llll}
\hline Name & Required & Data type & Description \\
\hline name & Required & aVF/aVR/aVL/I/II/II/VF/VR/VL & Stores the name of the limb lead. \\
\hline
\end{tabular}


Table 11: Description of the transformations element

\begin{tabular}{|c|c|c|c|}
\hline Elements & & & \\
\hline Name & Required & Data type & Description \\
\hline transformation & Required & See transformation table. & $\begin{array}{l}\text { This element, for example can } \\
\text { store equations for extracting } \\
\text { the } 12 \text {-lead ECG from the } \\
\text { BSPM. It can also be used to } \\
\text { transform one BSPM dataset } \\
\text { into another. }\end{array}$ \\
\hline
\end{tabular}

the lead element contains raw ECG values and the value calc indicates that the lead element contains an equation for deriving the lead. This is illustrated in Figure 11. Although, the type attribute is optional, if no value exists, then the value defaults to raw. Moreover, the type attribute should only be used if the type attribute in the bspm element has the value AVERAGED-BEATS-BSPMTRANSFORM or the value CONTINUOUS-BEATSBSPM-TRANSFORM.

\section{Results}

\section{Evaluation of the format}

This format has the potential to support the storage of all available BSPM datasets. It has been tested to store two BSPM datasets that have been widely reported in the literature. The Lux-192 dataset [32] consists of a $12 \times 16$ array of 192 leads (electrodes) as illustrated in Figure 12. Since all leads in a BSPM are unipolar, the terms lead and electrode may be used interchangeably. The rows in the Lux-192 layout are equally spaced between the suprasternal notch and the umbilicus. The columns are also equidistant around the whole thorax. Each lead contains a single averaged beat (approximately 600 sample values) sampled at $1000 \mathrm{~Hz}$. That equates to, approximately, 100, 000 data values in one file. This dataset has been stored using the proposed BSPM format. The file size was 293 kilobytes and was later compressed to 76 kilobytes using the ZIP compression algorithm. Also, the GZIP algorithm was used and was able to compress the file to just 68 kilobytes. As previously discussed, it can be said, that file size is not as important as it once was. However, telemedical systems may rely on small mobile devices, which rely on constrained processing environments and smart cards for storing data. In this context file size becomes very important.

Table 12: Description of the transformation element

\begin{tabular}{|c|c|c|c|}
\hline Attributes & & & \\
\hline Name & Required & Data type & Description \\
\hline name & Required & String & $\begin{array}{l}\text { This attribute stores the name } \\
\text { of the transformation dataset, } \\
\text { i.e. 12-lead ECG, VCG or BSPM. }\end{array}$ \\
\hline url & Optional & String & $\begin{array}{l}\text { This attribute retains a link to } \\
\text { an XML file containing the } \\
\text { calculations. }\end{array}$ \\
\hline \multicolumn{4}{|l|}{ Elements } \\
\hline Name & Required & Data type & Description \\
\hline transformLead & Required & See transformLead table. & $\begin{array}{l}\text { This stores an equation which } \\
\text { when executed calculates a } \\
\text { new lead. } \\
\text { e.g. }([\text { Lead52] + [Lead53])/2 }\end{array}$ \\
\hline
\end{tabular}


Table 13: Description of the transformLead element

\begin{tabular}{|c|c|c|c|}
\hline Attributes & & & \\
\hline Name & Required & Data type & Description \\
\hline name & Required & String & This attribute stores the name of the lead, e.g. aVF. \\
\hline $\boldsymbol{x}$ & Optional & Float & $\begin{array}{l}\text { Stores the } \times \text { axis for deriving the electrode position in } \\
\text { relation to the thoracic diagram. }\end{array}$ \\
\hline$y$ & Optional & Float & $\begin{array}{l}\text { Stores the } y \text { axis for deriving the electrode position in } \\
\text { relation to the thoracic diagram. }\end{array}$ \\
\hline location & Optional & $\mathrm{A} / \mathrm{P} / \mathrm{LL} / \mathrm{RL}$ & $\begin{array}{l}\text { Stores the general thoracic area of where the electrode } \\
\text { was attached. }\end{array}$ \\
\hline myocardialRegion & Optional & $\begin{array}{l}\text { An/HP/TP/IP/I/L/Ap/ } \\
\mathrm{RV} / \mathrm{S}\end{array}$ & Refers to the corresponding myocardial region. \\
\hline
\end{tabular}

Another BSPM dataset has been stored using this BSPM format, namely the Kornreich-117 lead set [26], which can be seen in Figure 13. The placement of electrodes in this layout is not as symmetrical as the Lux-192 lead set, i.e. the spacing between the columns in the Kornreich-117 is not equidistant. This BSPM dataset consists of 117 averaged beats (approximately 300 sample values) and was sampled at $500 \mathrm{~Hz}$. That equates to approximately 35,000 data values within one file. Unlike the Lux-192 dataset, the Kornreich-117 dataset retains three limb leads (VL, VR and VF) that were used to calculate the WCT. This means, a further 900 (approximately) data values have been stored within the limbLeads element. One Kornreich-117 dataset was stored at 257 kilobytes. This file was later reduced to 110 kilobytes using the ZIP compression algorithm. Alternatively, the GZIP

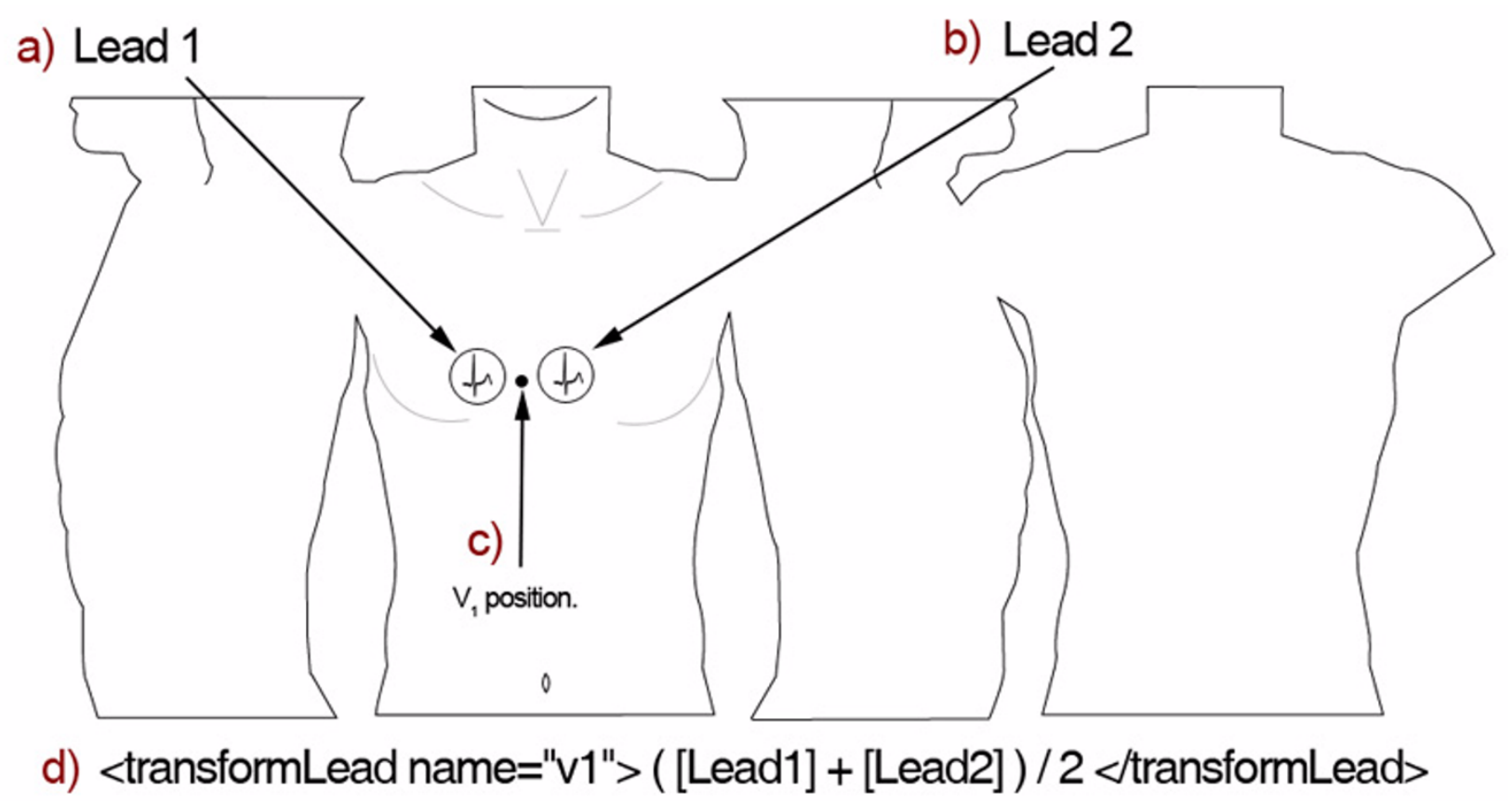

Figure 7 transformLead element. This diagram depicts how the transformLead element might be used to store a mean equation for calculating lead V1. 


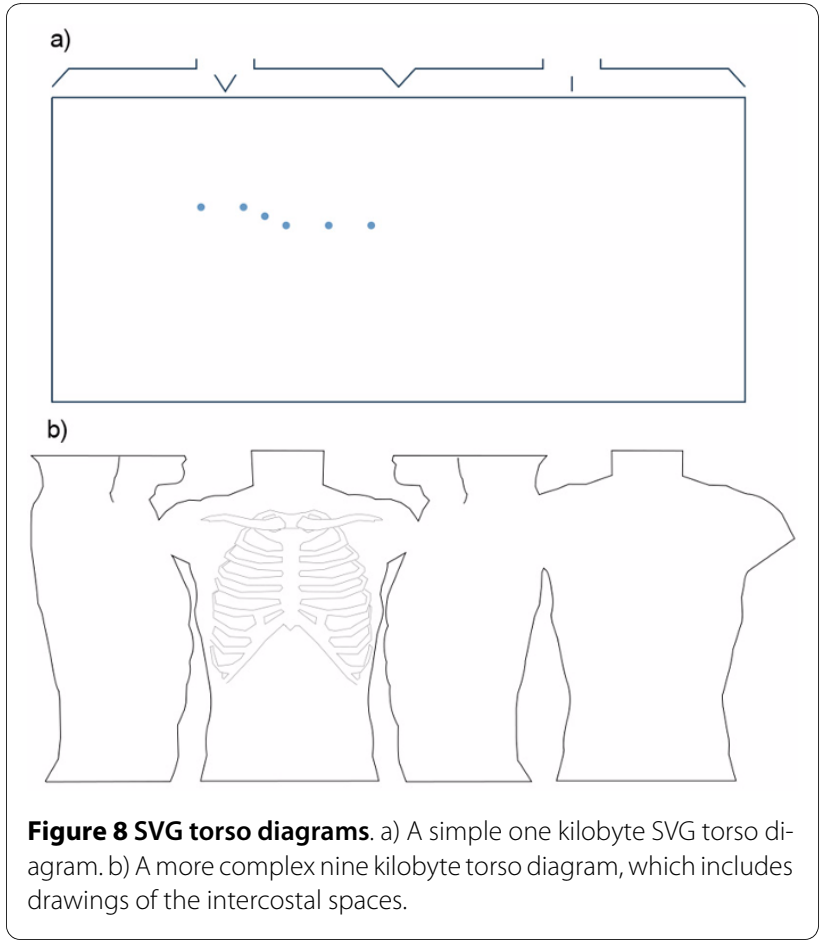

compression algorithm reduced the file to 111 kilobytes. These results have been summarised in Table 16 .

To truly evaluate this format and in particular, its file size, it would seem appropriate to compare these files with other BSPM files that have been formatted by a different standard. This is complicated by the fact that no other BSPM format exists. It would also be difficult to compare BSPM files with 12-lead ECG files that have been formatted with, for example SCP-ECG, since the storage of 8 leads and 200 leads are not comparable. Some 12-lead ECG files formatted using the aECG format can be as large as 500 kilobytes, which is a lot larger than the BSPM files that have just been evaluated within this study.

\section{Evaluation of the transformation feature}

Within the methods section of this paper, a technique was described for storing equations within the transformations element of the BSPM format. This method has been tested to define equations for extracting both a 12lead ECG and a VCG from a BSPM. A Lux-192 BSPM [32] was stored using this XML format. Figure 14 illustrates a study carried out by Drew et al. [33], which demonstrates the 12-lead ECG electrode positions in relation to the Lux-192 electrode positions. This diagram allowed us to determine the basic calculations required to extract a 12-lead ECG from a Lux-192 BSPM. The red circles indicate the 12-lead ECG electrode positions. When this particular dataset was recorded, the WCT was determined from standard (distal) limb leads. Unfortunately, this original limb lead data was not kept. As a result, the Mason and Likar (proximal) limb electrode positions have been used and yield the exercise variant of the 12lead ECG. Lead number 25 represents the right arm electrode, lead 85 represents the left arm electrode and lead 96 represents the left foot electrode. However, if the raw limb leads where kept and stored within the header of the format, such limb leads could have been used within these equations. This can be performed using the keyword "limbLead" concatenated with the name of the lead, i.e. "[limbLeadaVF]". As acknowledged, the Lux-192 dataset did not retain the original limb lead data, but can be obtained from the Mason and Likar torso leads. Moreover, the six precordial leads do not directly match any of the 192 leads. As a result, we define basic interpolative

Table 14: Description of the diagram element

\begin{tabular}{|c|c|c|c|}
\hline Attributes & & & \\
\hline Name & Required & Data type & Description \\
\hline url & Optional & String & $\begin{array}{l}\text { This attribute can store an } \\
\text { external link to an SVG } \\
\text { diagram, e.g.: http:// } \\
\text { www.raymondbond.com/ } \\
\text { bspmDiagram.svg }\end{array}$ \\
\hline waveScale & Optional & Float, DEFAULT: 0.04 & $\begin{array}{l}\text { This value is used to } \\
\text { proportionally scale the } \\
\text { waveforms in relation to the } \\
\text { size of the torso diagram, e.g.: } \\
\text { " } 0.04 \text { " scales the leads to } 4 \% \text { of } \\
\text { their actual size. }\end{array}$ \\
\hline
\end{tabular}




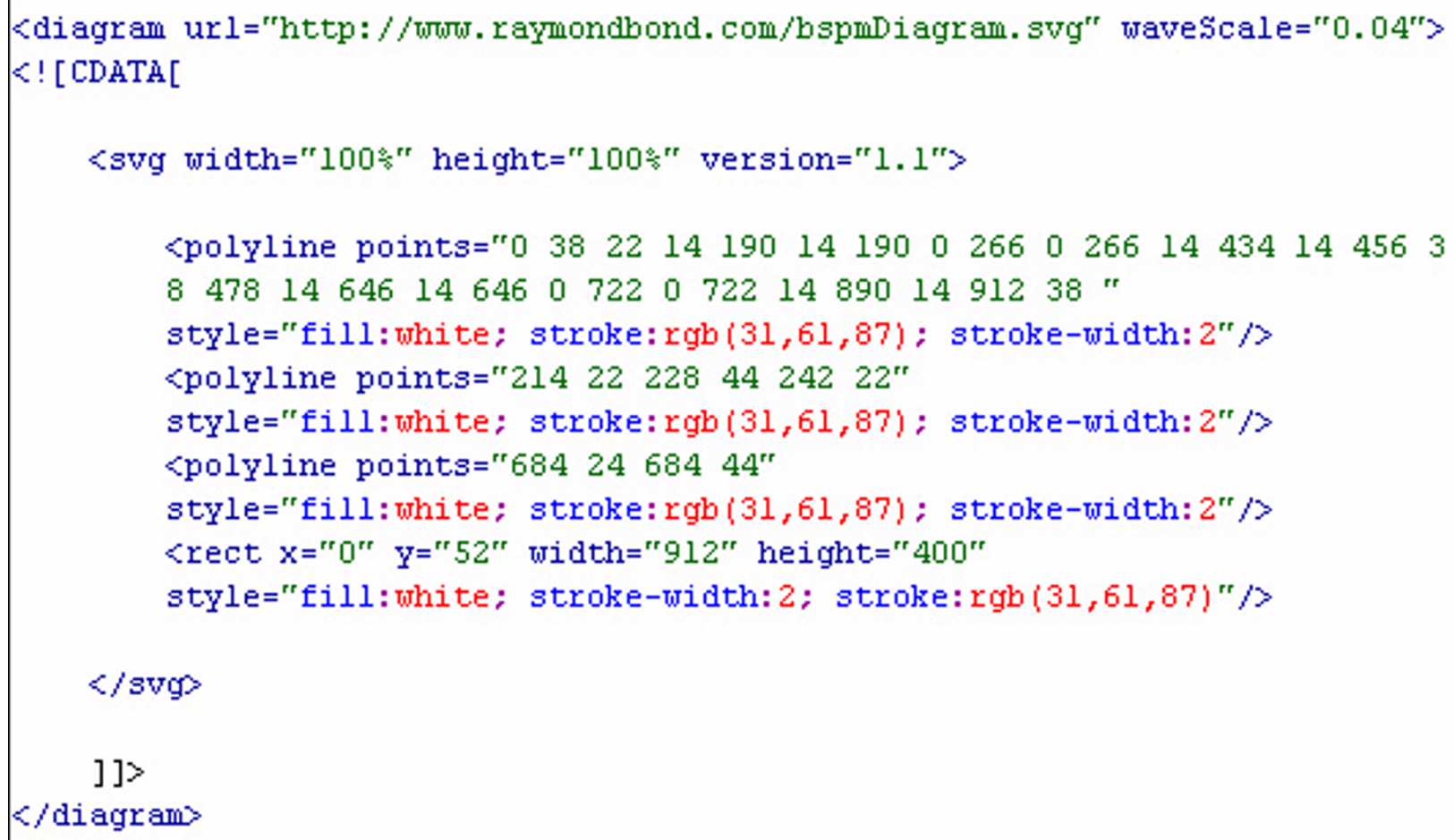

Figure 9 Embedded SVG diagram. This is an excerpt of the XML-BSPM format depicting the storage of the SVG diagram.

\section{Table 15: Description of the lead element}

\begin{tabular}{|c|c|c|c|}
\hline Attributes & & & \\
\hline Name & Required & Data type & Description \\
\hline Id & Required & Integer & $\begin{array}{l}\text { This integer is used to uniquely } \\
\text { identify and number each lead. }\end{array}$ \\
\hline$x$ & Required & Float & $\begin{array}{l}\text { Stores the } \times \text { axis for deriving the } \\
\text { electrode position in relation to the } \\
\text { thoracic diagram. }\end{array}$ \\
\hline$Y$ & Required & Float & $\begin{array}{l}\text { Stores the } y \text { axis for deriving the } \\
\text { electrode position in relation to the } \\
\text { thoracic diagram. }\end{array}$ \\
\hline Location & Optional & $\mathrm{A} / \mathrm{P} / \mathrm{LL} / \mathrm{RL}$ & $\begin{array}{l}\text { Stores the general thoracic area of } \\
\text { where the electrode was attached. }\end{array}$ \\
\hline myocardialRegion & Optional & $\mathrm{An} / \mathrm{HP} / \mathrm{TP} / \mathrm{IP} / \mathrm{I} / \mathrm{L} / \mathrm{Ap} / \mathrm{RV} / \mathrm{S}$ & $\begin{array}{l}\text { Refers to the corresponding } \\
\text { myocardial region. }\end{array}$ \\
\hline Data & Optional & raw/calc DEFAULT: 'raw' & $\begin{array}{l}\text { Defines whether the lead stores raw } \\
\text { data or a calculation. }\end{array}$ \\
\hline
\end{tabular}




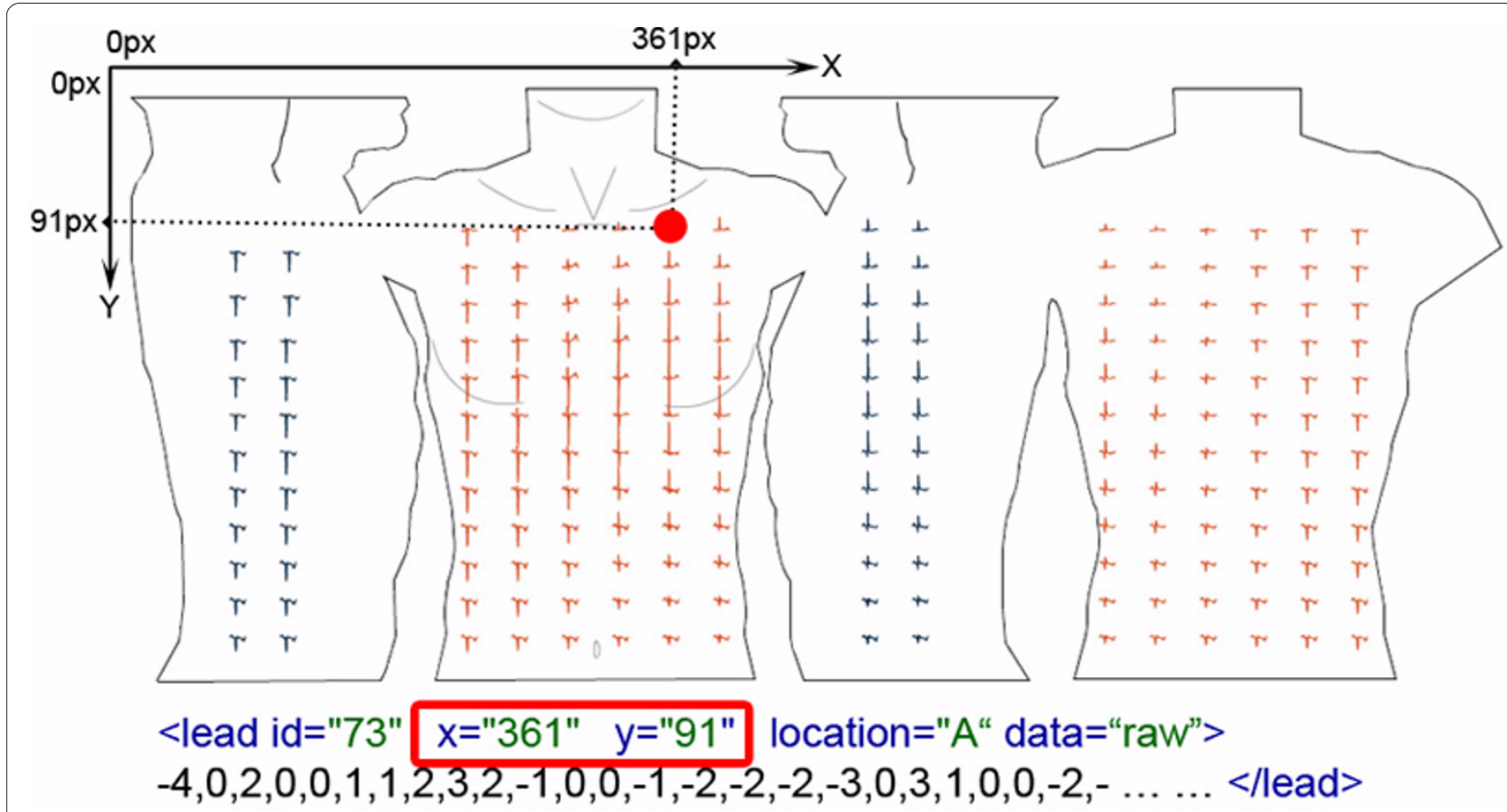

Figure 10 Storing electrode positions. This diagram illustrates a 2D coordinate system of how the attributes $x$ and $y$ are used to derive an electrode position.

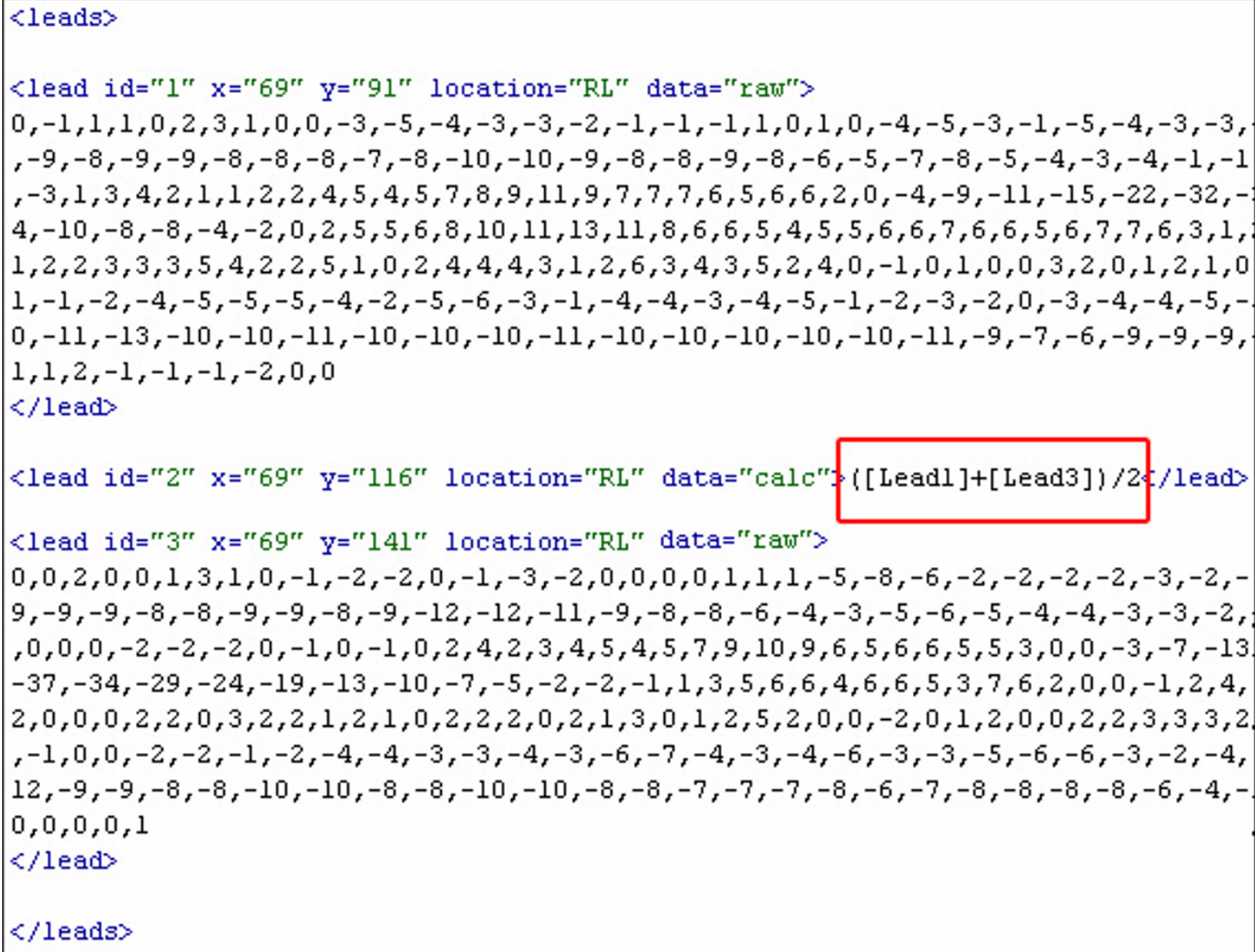

Figure 11 Calculated leads. This is an excerpt of the leads element where the type attribute has been used to distinguish between calculated leads and raw leads. 


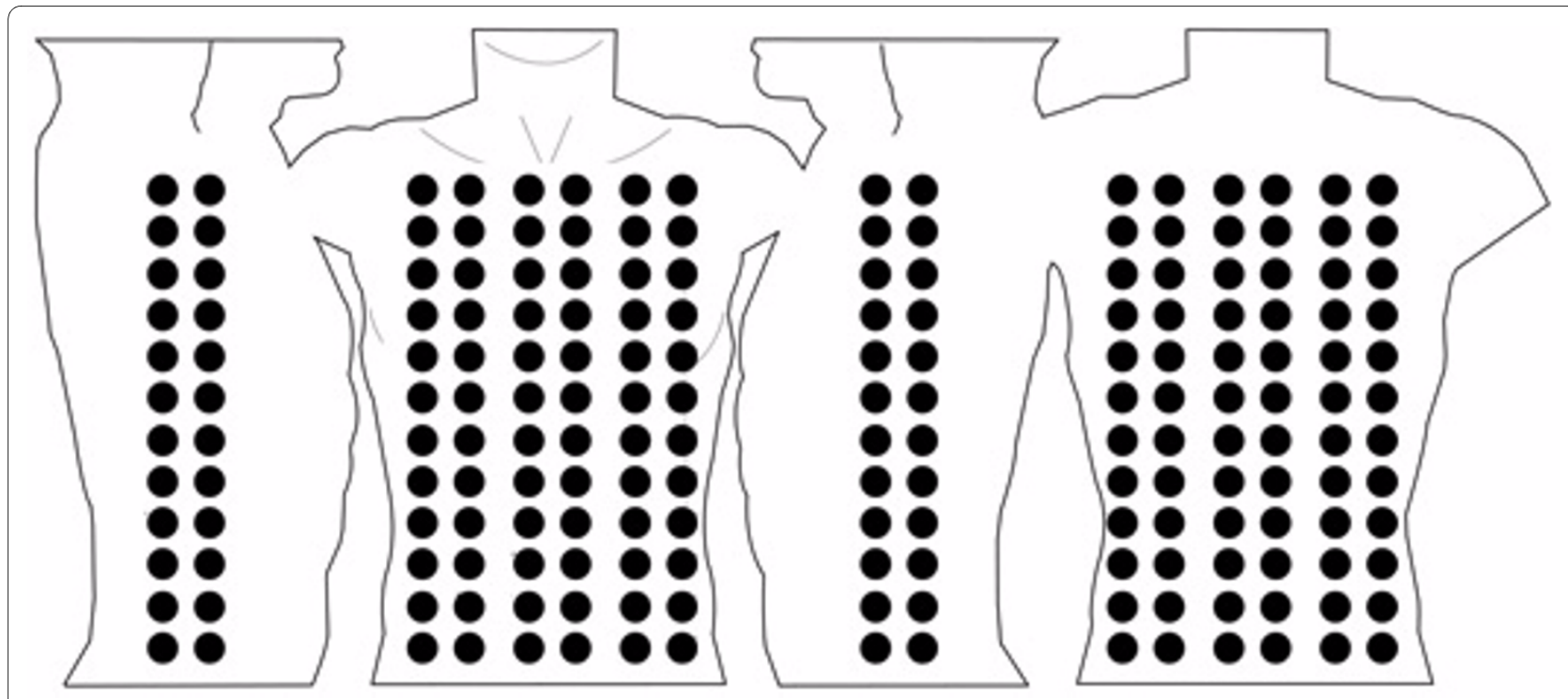

Figure 12 Lux-192 BSPM. Lux-192 electrode layout consisting of 192 electrodes equally spaced throughout a custom torso diagram.

Table 16: A comparison of BSPM file sizes when the file is stored as XML or compressed using either the ZIP or GZIP compression algorithms

\begin{tabular}{llll}
\hline BSPM dataset & Raw XML & ZIP compression & GZIP compression \\
\hline Lux-192 BSPM & 293 kilobytes & 76 kilobytes & 68 kilobytes \\
\hline Kornreich-117 BSPM & 257 kilobytes & 110 kilobytes & 111 kilobytes \\
\hline
\end{tabular}

calculations. For example, lead V1 would be positioned midway between leads 52 and 53 of the Lux-192 electrode layout. Therefore, a simple mean calculation as defined in Figure 14 can be used to calculate this lead.

As well as defining equations for deriving the 12-lead ECG, equations can also be defined to derive the VCG based on Frank lead system [34]. Figure 15 indicates the Frank based VCG electrode positions in relation to the Lux-192 layout [35]. Fortunately, all of the VCG recording sites are a subset of the BSPM electrodes. The green circles represent the $x$ axis, red circles represent the $y$ axis and the blue circles represent the $\mathrm{z}$ axis. Lead number 16 can be used as lead I and lead 100 can be used as lead A. The potential difference between leads I and A creates the $\times$ axis of the VCG. Lead 145 can be used as lead $H$ and lead 156 can be used as lead F. The potential difference between leads $\mathrm{H}$ and $\mathrm{F}$ make up the $\mathrm{y}$ axis. Finally, the $\mathrm{z}$ axis is the potential difference between lead 64 and lead 148 , better known as leads $\mathrm{E}$ and $\mathrm{M}$ in the context of VCGs. Figure 15 illustrates the calculations used to derive the $x, y$ and $z$ axes of the VCG. The 12-lead ECG and VCG equations can be seen in Figure 16. This figure shows how the equations look within the actual BSPM format.

\section{Discussion}

Accompanying tools

Since visual representation demonstrates a format, a web based BSPM viewer is currently underdevelopment for

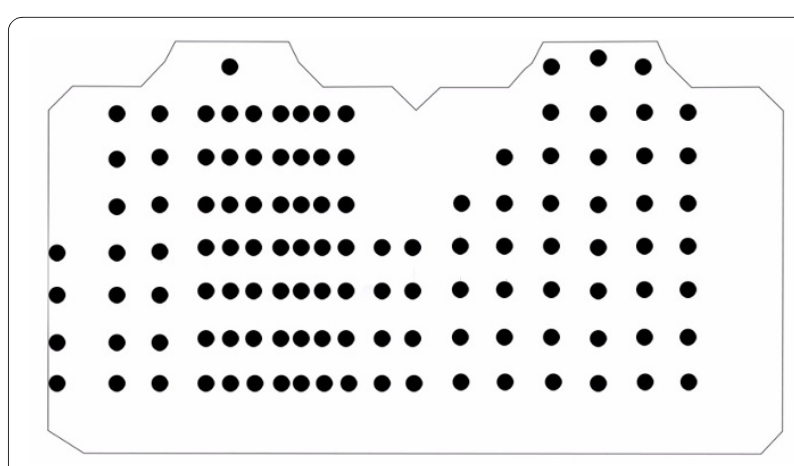

Figure 13 Kornreich-117 BSPM. Kornreich-117 electrode layout consisting of 117 electrodes placed throughout a custom torso diagram. 


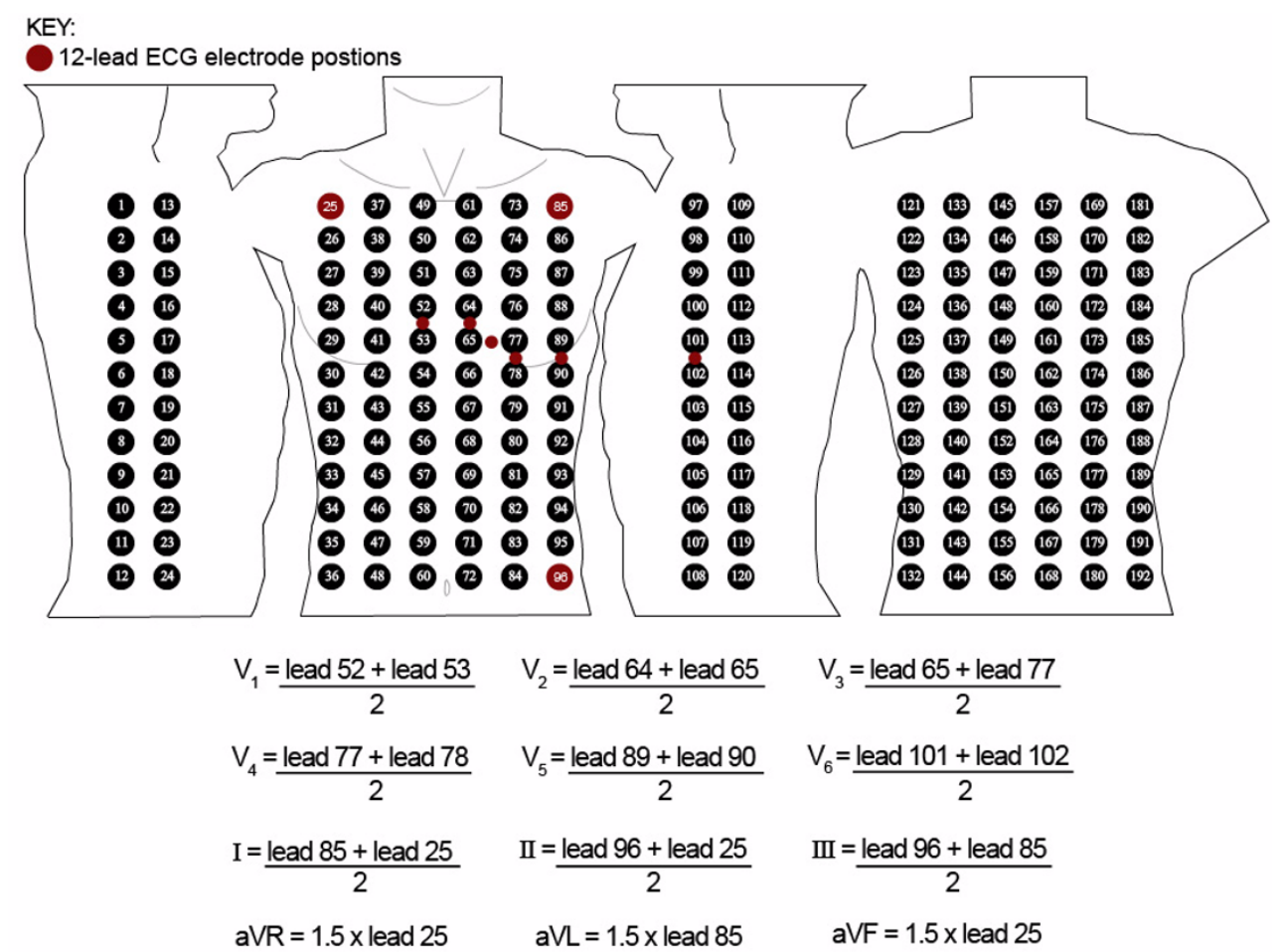

Figure 14 Extracting the 12-lead. The 12-lead ECG in relation to the Lux-192 BSPM and the basic equations used for extracting the 12 -lead ECG from the BSPM.

KEY:

xaxis yaxis zaxis

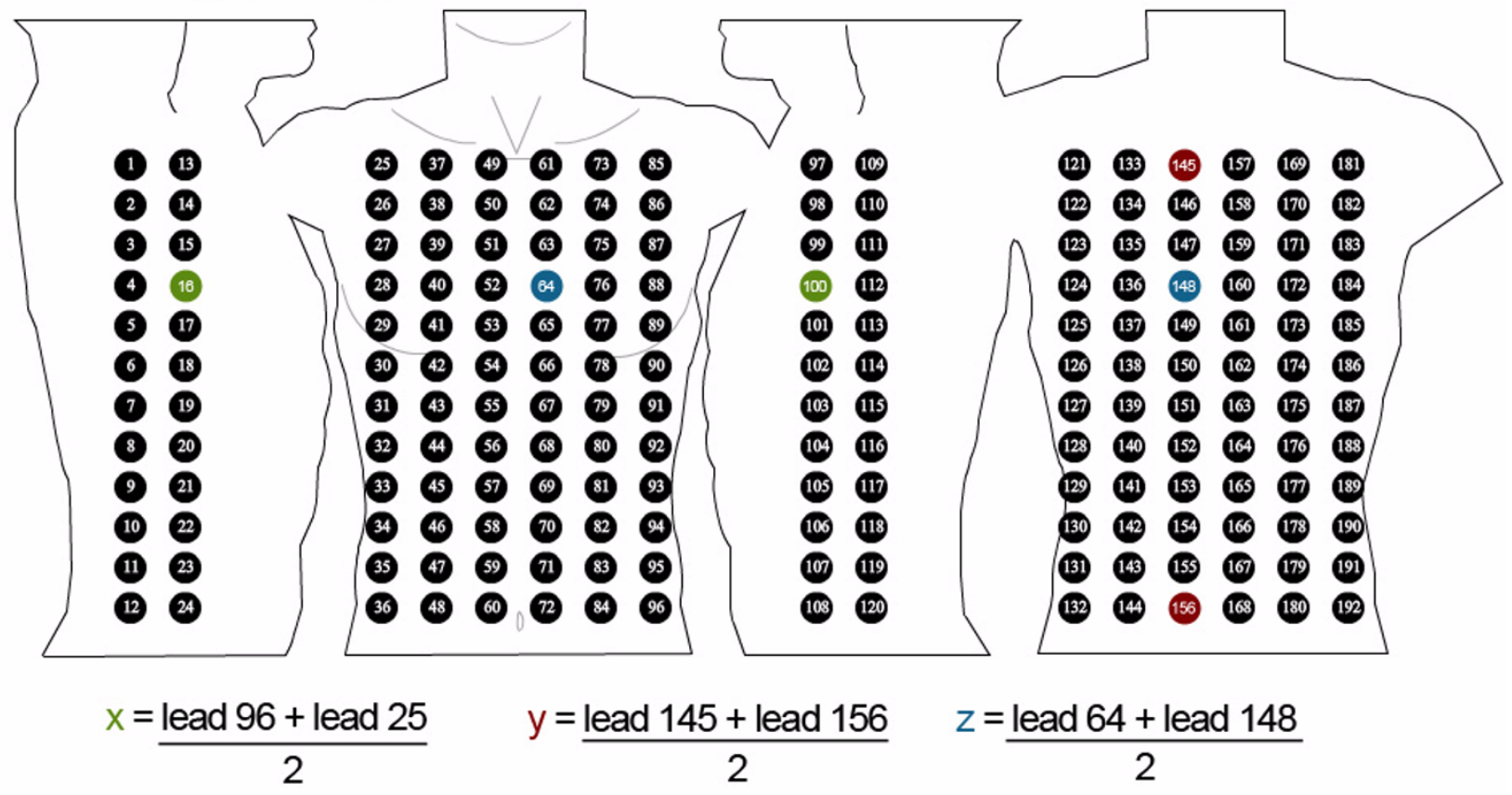

Figure 15 Extracting the VCG. This diagram highlights the leads from the Lux-192 BSPM and the equations used to calculate the VCG. 


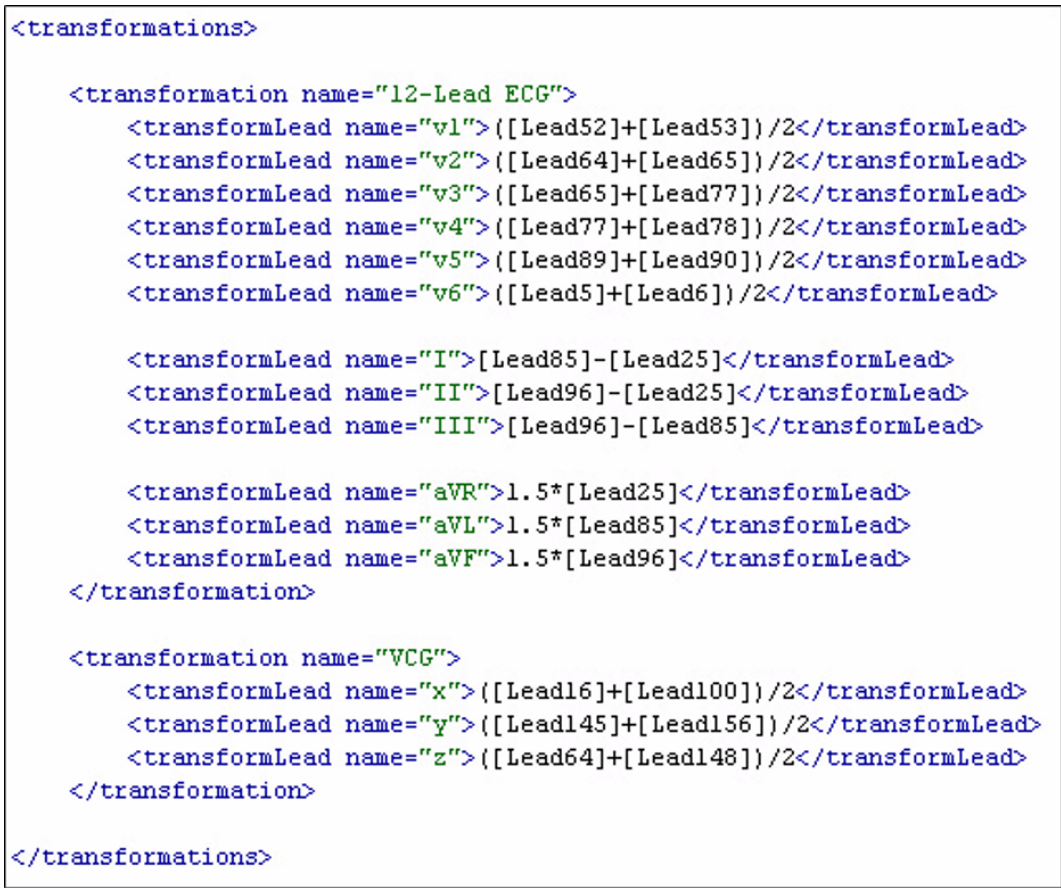

Figure 16 12-lead ECG and VCG equations. This diagram contains the 12-lead ECG and the VCG equations as defined within the XML-BSPM format.

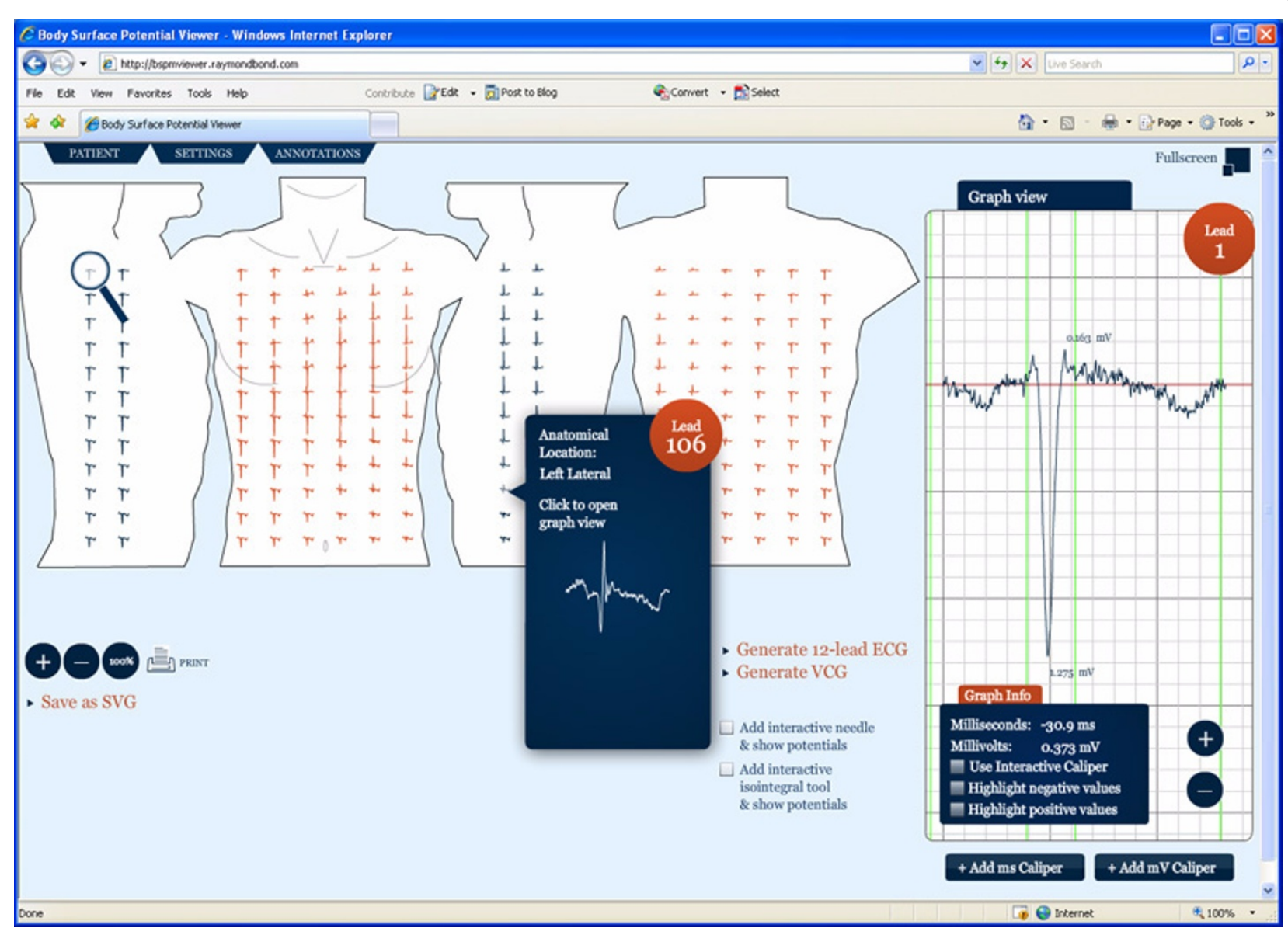

Figure 17 BSPM viewer. A web based BSPM viewer for parsing, processing and visualising XML-BSPM files. 
staging the capabilities of this BSPM format. The current version of the system can be found online [36]. This Rich Internet Application (RIA) parses the format, renders the SVG torso diagram, draws the BSPM leads and provides interactive tools to allow clinicians to intuitively explore the BSPM. These tools include callipers, an isopotential and an isointegral tool. This BSPM viewer was created using the Adobe Flash technology [37]. This application is geared towards both the clinician and the engineer, whereas traditional BSPM tools were designed only for the engineer, i.e. Map3D [38]. A screenshot of the BSPM viewer can be seen in Figure 17.

\section{Future work}

The format proposed in this paper has been developed to the level where it is useable. However, a number of additional tests are foreseen to prove the full utility of the format. These tests include the ability to securely store long term/continuous data as opposed to averaged beats and the ability to support data recorded from limited lead sets. Further work will also include the completion of the web based BSPM viewer, a format validation service, a BSPM to SVG converter and possibly an online warehouse, where users can share and download BSPMs. In summary, this research provides initial ground work for creating a complete BSPM management system.

\section{Conclusions}

The work presented in this paper documents one of the first attempts to establish a storage format that supports data recorded from any BSPM recording configuration. The primary goal has been to promote the storage and sharing of data that traditionally has not been widely accessible. Although the format has been conceived, bearing in mind the requirements of storing data recorded from a large number of leads, it can in fact be used to support data from any ECG recording. That said, this proposed format should not be seen as a competitor to well established formats such as SCP-ECG, DICOM and aECG. The lessons learned in this work and reported in this paper can be used for the further development of existing ECG formats and standards; which in the future may be enhanced to support BSPM leads.

\section{Competing interests}

The authors declare that they have no competing interests.

\section{Authors' contributions}

RRB carried out the literature review, designed the XML schema for storing BSPMs and drafted this manuscript. DDF, CDN and GM participated in the coordination and design of this study and also helped draft this manuscript. All authors read and approved the final paper.

\section{Acknowledgements}

The authors would like to acknowledge Professor Robert Lux and Professor Fred Kornreich for provision of BSPM data.

\section{Author Details}

Computer Science Research Institute, School of Computing and Mathematics, University of Ulster, Shore Road, Newtownabbey, Co. Antrim, BT37 0QB, UK

Received: 15 February 2010 Accepted: 14 May 2010

Published: 14 May 2010

\section{References}

1. Carley SD, Jenkins $M$, Jones KM: Body surface mapping versus the standard 12 lead ECG in the detection of myocardial infarction amongst emergency department patients: a Bayesian approach. Resuscitation 2005, 64(3):309-314.

2. McClelland AJJ, Owens CG, Menown IBA, Lown M, Adgey AAJ: Comparison of the 80-lead body surface map to physician and to 12lead electrocardiogram in detection of acute myocardial infarction. Am J Cardiol 2003, 92(3):252-257.

3. LuX RL: Uncertainty of the electrocardiogram: old and new ideas for assessment and interpretation. J Electrocardio/ 2000, 33(Suppl):203-208.

4. Sfakianakis S, Chronaki CE, Chiarugi F, Conforti F, Katehakis DG: Reflections on the role of open source in health information system interoperability. Yearb Med Inform 2007:50-60.

5. Chronaki CE, Chiarugi F, Macerata A, Conforti F, Voss H, Johansen I, RuizFernandez R, Zywietz C: Interoperability in digital electrocardiography after the openECG project. Proceedings of IEEE Computers in Cardiology 2004:49-52.

6. Fischer R, Chiarugi F, Zywietz TK: Enhanced integrated format and content checking for processing of SCP ECG records. In Proceedings of IEEE Computers in Cardiology 2006:413-416.

7. Wang H, Azuaje F, Jung B, Black N: A markup language for electrocardiogram data acquisition and analysis (ecgML). BMC Medical Informatics and Decision Making 2003, 3(1):4.

8. Lu X, Duan H, Zheng H: XML-ECG: An XML-based ECG presentation for data exchanging. In Proceedings of Bioinformatics and Biomedical Engineering ICBBE 2007:1141-1144.

9. ECG description in MFER and HL7 version 3 [http://kosmi.snubi.org/ 2003 fall/APAMI CJKMI/O8-4-023-Hirai-0829.pdf

10. Mildenberger P, Eichelberg M, Martin E: Introduction to DICOM. European radiology 2002, 12(4):920-927.

11. Dicom.offis.de - general information - introduction to the DICOM standard [http://dicom.offis.de/demintro.php.en]

12. Hilbel T, Brown BD, de Bie J, Lux RL, Katus HA: Innovation and advantage of the DICOM ECG standard for viewing, interchange and permanent archiving of the diagnostic electrocardiogram. In In Proceedings of IEEE Computers in Cardiology Conference Durham; 2007:633-636.

13. HL7 aECG implementation guide [http://www.amps-Ilc.com/ UsefulDocs/aECG Implementation Guide.pdf]

14. Chronaki CE, Chiarugi F, Sfakianakis S, Zywietz C: A web service for conformance testing of ECG records to the SPC-ECG standard. In In Proceedings of IEEE Computers in Cardiology Conference Lyon; 2005:961-964.

15. de Wijs MCJ, van Ettinger M, Meij SH, Nelwan SP: Integration of multiple ECG databases into a unified framework. In In Proceedings of IEEE Computers in Cardiology Conference Lyon; 2005:447-450.

16. Hoekema R, Uijen GJH, van Oosterom A: On selecting a body surface mapping procedure. Journal of Electrocardiology 1999, 32(2):93-101.

17. Joint recommendations of the American Heart Association and the Cardiac Society of Great Britain and Ireland: Standardization of precordial leads. Am Heart J 1938, 15:107-108.

18. Hampton JR: The ECG made easy. 6th edition. Edinburgh; New York: Churchill Livingstone; 2003.

19. How to implement SCP - part I [http://www.openecg.net/tutorial1/ index.html]

20. ECGAWARE An ECG markup language for ambulatory telemonitoring and decision making support [http://www.inf.ufes.br/ bgoncalves/ contents/healthinf08.pdf]

21. Fang Q, Sufi F, Cosic I: A Mobile Device Based ECG Analysis System. In Data Mining in Medical and Biological Research 2008:209-225. IN-TECH

22. Ng W, Lam W, Cheng J: Comparative Analysis of XML Compression Technologies. World Wide Web 2006, 9(1):5-33.

23. How XML is improving data exchange in healthcare [http:// www.softwareag.com/xml/library/schroeter healthcare.htm]

24. FDA XML Data Format Design Specification [http:// xml.coverpages.org/FDA-EGC-XMLDataFormat-C.pdf] 
25. Donnelly MP, Nugent CD, Finlay DD, Black ND: Diagnostic assessment of transformation of body surface potential mapping lead systems. J Electrocardio/ 2006, 39(4, Supplement 1):S31-S32.

26. Montague TJ, Smith ER, Cameron DA, Rautaharju PM, Klassen GA, Felmington CS, Horacek BM: Isointegral analysis of body surface maps: surface distribution and temporal variability in normal subjects. Circulation 1981, 63(5):1166-1172.

27. Horácek BM, Warren JW, Feild DQ, Feldman CL: Statistical and deterministic approaches to designing transformations of electrocardiographic leads. J Electrocardiol 2002, 35(4, Part 2):41-52.

28. Robinson MR, Curzen N: Electrocardiographic Body Surface Mapping: Potential Tool for the Detection of Transient Myocardial Ischemia in the 21st Century? Annals of Noninvasive Electrocardiology 2009, 14(2):201-210.

29. Specification of the EBS file format for bio-signals [http:// www.cl.cam.ac.uk/ mgk25/ebs/]

30. Scalable vector graphics [http://www.w3.org/Graphics/SVG/

31. Varni A, Kemp B, Penzel T, Schlogl A: Standards for biomedical signal databases. IEEE Eng Med Biol 2001, 20(3):33-37.

32. Lux RL, Smith CR, Wyatt RF, Abildskov JA: Limited Lead Selection for Estimation of Body Surface Potential Maps in Electrocardiography. IEEE Transactions on Biomedical Engineering 1978, 25(3):270-276.

33. Drew BJ, Schindler DM, Zegre JK, Fleischmann KE, Lux RL: Estimated body surface potential maps in emergency department patients with unrecognized transient myocardial ischemia. J Electrocardiol 2007, 40(6S1):15-20.

34. Frank E: An accurate, clinically practical system for spatial vectorcardiography. Circulation 1956, 13(5):737-749.

35. ECG measurement and analysis [http://www.cvrti.utah.edu/ macleod/ bioen/be6000/labnotes/ecg/descrip.html]

36. Body surface potential map viewer [http://bspm.raymondbond.com/

37. Adobe flash player [http://www.adobe.com/products/flashplayer/]

38. MacLeod RS, Johnson CR: Map3d: Interactive scientific visualization for bioengineering data. In Proceedings of the 15th International Conference on Engineering in Medicine and Biology Society 1993:30-31.

\section{Pre-publication history}

The pre-publication history for this paper can be accessed here: http://www.biomedcentral.com/1472-6947/10/28/prepub

doi: $10.1186 / 1472-6947-10-28$

Cite this article as: Bond et al., XML-BSPM: an XML format for storing Body Surface Potential Map recordings BMC Medical Informatics and Decision Making 2010, 10:28

Submit your next manuscript to BioMed Centra and take full advantage of:

- Convenient online submission

- Thorough peer review

- No space constraints or color figure charges

- Immediate publication on acceptance

- Inclusion in PubMed, CAS, Scopus and Google Scholar

- Research which is freely available for redistribution

Submit your manuscript at www.biomedcentral.com/submit
C Biomed Central 